\title{
AXISYMMETRIC BUCKLING OF ANNULAR SANDWICH PANELS
}

\author{
By \\ AMELIO JOHN AMATO
}

\begin{abstract}
A DISSERTATION PRESENTED TO THE GRADUATE COUNCIL OF THE UNIVERSITY OF FLORIDA

IN PARTIAL FULFILLMENT OF THE REQUIREMENTS FOR THE DEGREE OF DOCTOR OF PHILOSOPHY
\end{abstract}

UNIVERSITY OF FLORIDA 


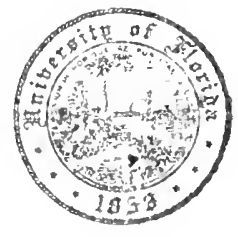

UNIVERSITY OF FLORIDA

31262085522943 
To my wife,

Carol 


\section{ACKNOWIJDGMENTS}

The author wishes to express his appreciation to the members of his evpervisory comittee: to Dr. 1. K. Ebcioglu, chaiman, who criginally suggested the topic and, through his constant guidance and encouragenent, made this work possible; to Drs. S. Y. IM, V. H. Kurzwes, N. A. Ejsenberg, and k. H. Hadlock for theix helpful chiscussions with the author and many valuabje siggestions.

The alithor also wishes to express his thanls to the NDEA Title IV progran for the financial support accorded to him.

Finally, the author wishes to thank his wife, carol, without Whose patience, understandjng and encouragenent he would not have been able to complete this task. 
TABLE: OF CONTENTS

Page

AChNOHLDGMTTS ..................... i i

LIST OF TABLES . . . . . . . . . . . . . . . . . . . vi vi

LIS Of FJGLite . . . . . . . . . . . . . . . . . . vii

KEY TO SYMUTS . . . . . . . . . . . . . . . . . . viii

ABSTUACT . . . . . . . . . . . . . . . . . . . . $\mathrm{xi}$

CIINTLR

I. Introdiction . . . . . . . . . . . . . 1

II. BASIC CONCETSS . . . . . . . . . . . . . 6

1. Description of Sandwich Panel and Notations . . . 6

2. Displacements . . . . . . . . . . . . 8

3. Strain-Injsplacement Rolations . . . . . . . . . . . 9

4. Stress-Strain Relations . . . . . . . . . . 10

5. Stress Resultants . . . . . . . . . . 12

I1. DTRIWIIOY OF EQUATONS IN CARTESIAN COORDINATTS . • . 14

1. Total Potential .. . . . . . . . . . Id

2. Theorem of kininum Potential Energy . . . . . . . 16

3. Equilibrium Equations and Boundary Conritions . . I9

4. Simplification of Equations . . . . . . . . . 22

IV. FQU? LIBRIUM EQUATIONS AND BOUNDARY CONDIJIGNS

IN CSLINDRICAL COORDINATES . . . . . . . . . . . 25

1. Applicalion of Covariant Derivatives. . . . . . 25

2. Transforliation of Equations . . . . . . . . 30

3. Axial Stress Distribution . . . . . . . . . 32

4. Reduced Equilibrium Equatinns . . . . . . . . 35

5. Bomciary Conditions . . . . . . . . . . . 38

6. Comparjson with Othej Thenjes . . . . . . . . 39 
TABLE OF CONTENTS (Continued)

CHA'J'TR

Page

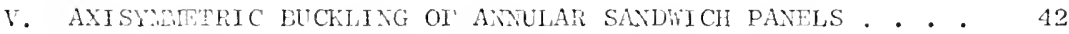

1. Lniform Axjal Stress Distribution . . . . . . 42

2. General Solution. . . . . . . . . . . . 46

3. Successire Approximations . . . . . . . . . 53

4. Runerical Results and lliscussions . . . . . . . . c co

5. Error Bown . . . . . . . . . . . . . . 66

6. Remarts on Contrgence ............. 68

li. CONCblsion. . . . . . . . . . . . . . . 7 . .

BIDIIOGRIIII . . . . . . . . . . . . . . . . . . . 73

BIOGRSTILCHL SKETCH . . . . . . . . . . . . . . . . 75 


\section{LIST OE TABLES}

Tab]e

Page

1. Comparison of Equations Governing Stability of SingleJayer Panels and Sandwich Panels . . . . . . . . . . .

2. Lowest Value of $\mu$ Satisfying lquation (86) . . . . . . .

3. Approzimate values of $\left(\mathrm{x}_{\mathrm{O}} / \mathrm{A}\right)_{\mathrm{Cr}}$ for $\equiv=0$. . . . . . . 


\section{LIST OF FIGURES}

Figure

Page

1. Element of Sandwich Panel . . . . . . . . . . . 7

2. Anmular Sandwich Panel . . . . . . . . . . . . . 34

3. Minimum Critical Values of $\left(\mathrm{N}_{\mathrm{o}} / \mathrm{A}\right)$ for $\mathrm{K}_{\mathrm{O}}=\mathrm{N}_{\mathrm{i}} \cdot \cdot \cdot \cdot 63$

4. Minimum Critical vaiues of $\left(N_{0} / A\right)$ for $N_{i}=0$. . . 64

5. Radius of Convergence . . . . . . . . . . . . . . . 68 


\section{KEY TO SYMBOLS}

$$
\begin{aligned}
& V^{\prime}, V_{3} \\
& =\text { Displacements } \\
& \text { "), 'c, w } \\
& =\text { Displacement components } \\
& x, y, z \\
& \text { = Cartesian coordinates } \\
& i^{\prime}, z^{\prime} \\
& \text { = Coordinates, defined by equation (1) } \\
& t_{c}, t_{f} \\
& \text { = Thicknesses of core and faces, respectively } \\
& =\text { Superscrjpts indicating the lower and mper. } \\
& \text { face quantities, respectively } \\
& \hat{t} \quad=\left(t_{c}+t_{f}\right) / t c \\
& \text { ir } \\
& \text { - Total potertial } \\
& \mathrm{U}_{\mathrm{c}}, \mathrm{u}_{\mathrm{f}} \\
& =\text { Strain encrgic: }
\end{aligned}
$$

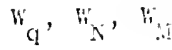

$$
\begin{aligned}
& =\text { Worr performed by external forces and moments } \\
& \text { a, E, Y, H } \\
& =\text { Indices taling on values } x \text { or } y \\
& \mathrm{j}, \mathrm{j}, \mathrm{k} \\
& =\text { Inrijees taking on talues } x, y \text { or } z \\
& \epsilon_{i j}, e_{2,}, e_{\alpha 3}, e_{33}=\text { Components of strain }
\end{aligned}
$$

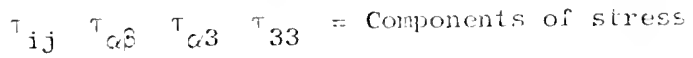

$$
\begin{aligned}
& \mathrm{G}_{\mathrm{C}} \\
& v_{1} \\
& \text { Iif } \\
& n_{f} \\
& \text { A } 0,3 y \\
& \delta<? \\
& \underline{\mathrm{N}}-\mathrm{a} \\
& \text { = Snear modulus of tie coìe }
\end{aligned}
$$

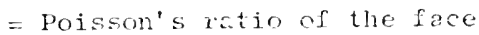

$$
\begin{aligned}
& \text { - Young's modulus of the race } \\
& \text { = Bending rigidily of the face }
\end{aligned}
$$

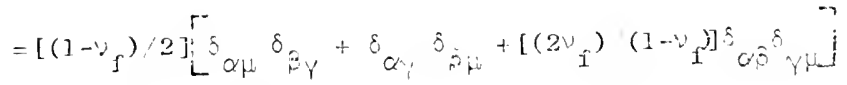

$$
\begin{aligned}
& \text { = Kronectier delia }
\end{aligned}
$$

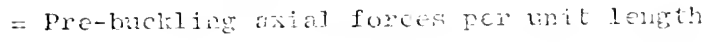


$\frac{M}{-\alpha}$

$Q_{\alpha}$

$M_{\alpha \beta}$

$N_{\alpha}$

(n)

$\left\{\begin{array}{c}2 \\ n a\end{array}\right\}$

$x^{4}$

$n, \ell, c, s, t, m, p$

$\mathrm{g}^{\mathrm{lm}}, \mathrm{g}_{\mathrm{lm}}$

$\mathrm{X}_{\mathrm{n}}, \mathrm{Y}_{\mathrm{n}}^{\mathrm{s}}$

$r, \theta$

$\nabla^{2}$

$\bar{u}$

$\mathrm{u}$

$\mathrm{N}_{i}, \mathrm{~N}_{0}$

a, b

?

D, E

$\varphi$

F, G, H, I
= Lateral load per unit area

= Externally applied monent per unit length

$=$ Transverse shear forces per unit length

$=$ Bending moments per unit length

= Axial forces per unit length corresponding to snall cleformations cluring buckling

= Bracleted index indicating physical component of a tensor

$=$ Christoffel symbol of the second kind

$=$ General coorinate variable

$=$ Indices taking on values 1,2 and 3

$=$ Metric tensor

= Arbitrary tensors

$=$ Polar coorclinaics

= Laplacian operator

$=$ pre-buckling lateral displacement of middle plane of faces

$=$ Iateral displacement of micidle plane of faces for axisymetric equations

= Compressive axias forces per unit longth applied to imner and outer edges, respectively

= Inner anci outer radij of annular panel, respectively

$=\mathrm{a} / \mathrm{b}$

= Defined by cquation (65)

$=\frac{d w}{d r}$

= Defined by equation (72) 


\begin{tabular}{|c|c|}
\hline$A, B$ & $=$ Defined by equation (73) \\
\hline$\eta$ & $=\mathrm{r} / \mathrm{b}$ \\
\hline$N, R, Q$ & $=$ Defined by equation $(9 \hat{A})$ \\
\hline$\xi$ & $=n-1$ \\
\hline$A_{k}$ & $=$ Coefficients of power series \\
\hline$\mu$ & $\begin{aligned}= & \text { Buckling coefficiont for uniform axial } \\
& \text { stress distribution }\end{aligned}$ \\
\hline $\mathrm{C}_{1}$ & $=$ Arbitrary constant of integration \\
\hline$J_{1}, Y_{1}$ & $=$ Bessel functions of order one \\
\hline & $=$ Radius of convergence \\
\hline
\end{tabular}


Abstract of Dissertation Presented to the

Graduate Council of the University of Florida in partial Fulfillment

of the Requirments for the Dezree of Doctor of Philosolny

AXI SYAIETRIC BUCKLING OF ANNULAR

SATDWICH PANEIS

By

Amelio John Anato

June, 1970

Chaiman: Dr. Jbrahim K. Ebcjornu

Major Department: Enginecring Scjence and Mechanics

The buckling of an annular sandwich panel is investigated

using the theorern of minimum jotential energy. Governing equations,

derived in cartesian coordinates, are transformed into cylindrical

corrdinates by meons of covarjant differentiation. Considering the

faces to be nembranes and assuning an axisymetric buckling mode, the equilibrium equations axe uncoupled through the application of an improver technigue.

For a clamped outer eige and "slider" inmer edge a series solutjon is applied to the reneral probler of radially varying intplare stresses. Critjcal stresses are plotted versus ratio of inner and outer radij. The fjfth approximation is shown to yield acceptable rosults. 


\section{CHAPTER I}

\section{INTRODUC'TION}

A sandwich panel is defined as a three-layer panel, consisting of two thin onter layers of high-strength material between which a thick layer of low avorage strength and donsity is sandwiched. The two thin onter Jayers are called faces, and the internodiate lajer is the core of the pane] [1].

Among the main advantages of sandwich construction are: a high rigidity to weight ratio, good themal and accoustical insulntion, and ease of mass production. Some examples of core materials are balsa wood, cellulose acetate and synthetjc rubber. Honever, in more recut times thin foils in the form of hexagonal cells porpendicular to the faces have been employed. Depending upon the intended application, faces may be constructed of alumjum alloys, high-stiength stecl, ete.

The practicai importance of sandwich construction came into prominence with tie advent of the aircraft and suace industries. With the need for lighter, stronger and more stable structural com.ponents, great emphasis was placed upon the design and ansysis of workable sandwich pancls.

\footnotetext{
* Numbers ir; hrackets refer io the Bibliograply.
} 
The essential differcuce between the analysis of single-layer panels and that of sandwich panels is that the shear deformation associatcd with the core of a sandwich panel may not be neglected. Moreover, initially julne sections no longer remain plane during bending, and the existing plate theories $[2,3]$ require extensive modifications.

Numerous authors have contributed to the development of mathematical theories describing the behavior of rectengular sandwich pancls. Two of the more noted of these are Hoff [4] and Eringen [5] who, carly in the 1950's illustrated a concise and straightforward approach to the problem using the thooren of minimu potential energy. In 1960 Crang and Fbcioglu [G] introduced continuity conditions for displacements across the interface of two adjacent layers. This modification has been shom [7] to contribute aprecjably to the accuracy of the cicrived equations, whle introcheing no additional mathematical complications.

In comprison, circular sandwich pancls have been the subject of relatively little investigation. In 1919 Eric keissner [8], negleeting the bending rigidity of the faces, solved the problem of a circular serciwich panel subjected to axisymetric transterse loading. Later Zajd [9] inclucled the effeets of the bending rigidity of the face layers. Huang and Jbcioglu [10], using a technique sinilar to that cmployed by zaje, Jeecontly jnvestigoted the axjsymetric buckling of a circular sandwich pancl subjected to uniform axjal compression. In their find results, the faces were treated as mombanes. 
Prior to the arvent of sandwich construction, the stability of circular and anmular single-layer panejs were extensively juvestigated [3]. Mejssner [1.1] analyzed the axisymetric bucliling of a sing]elayer anmular panel subjected to uniform compression alung the outer bounclary. In this rork, the inner boundary was consiciered iree and the outer boundary simply supported or clamped. Olsson [12] extended Neissner's annysis by considering the outer boundary clomped and the inner boumdary "slider." Such a condition coud be approximated by allowing a shaft or rigic cyinder to occupy the central hole (see Figure 2).

Prompted by the foregoing secuence of investigations, the main objectives of the present malysis are: (i) to paraldel the sceucence of analysis present in the literature of single-layer panels by inves. tigating the axisymetric bucling of amular sanciwich panels; (ji) to achieve a nore satisfactory fommation of the theory through the use of continuity conclitions [6]; (iii) to modify the uncoupling proceciure introduced by ziajd [9] ond later employed by Ihang and Ebeioglu [10], thereby signiricantly raducing the complexity of the uncoupled rauilibrim equations; and (iv) to apply the boundary conditions employed by Olsion [12] to the present work.

For the initiol derivation, the set of basic assumptions employed in the present analysis are:

(A1) The effect of transverse normal stress in each layer is negligible.

(A2) The core unciergocs shear clefomation only.

(A.3) Displacencinte in wath lay ax linotr functions of distence from the nedian plane of the layer. 
(A4) The median planc of the core remains neutral during small deflections of the panel.

(A5) Fach layer is homogeneous and isctropic.

(A6) The core is attached to the faces securely.

(A7) llooke's law is valicl throughout.

(As) Local buckling of the pancl does not occur.

These assumptions are similar to those usej by hoff [4] and Chang and Ebcioglu [13].

Equilibrium equations and boundary conditions are durved, using the above assunptions and the theoren of minimum potential enery. These equations are then transfornd inte polar coordinates through the application of tensor analysis. In order to solve our problem, the resulting equations are then simplified further using the following aditional assumptions:

(131) Berromli-Narier hypothesis is valid for the faces.

(132) Faces are considered to be nembrancs.

(L3) Anular sandwich panel subjected to uniform axial compression bucliles axisymetrically.

The first assumption is valid whon the shear deformation of tite face laycrs is negligible. Kim [7] has show this to be true for most practical applications. The second assumption, which has becn employed by many authors [S,J0], is discussed at length by Plantema []]. Finally, the assumution of axisymetric buckling cannot be rignously vorified without solving the more difficull problem of unsymetric buesting. Howevor, such an assumption wont a seon reasonald since olsson [12] haz

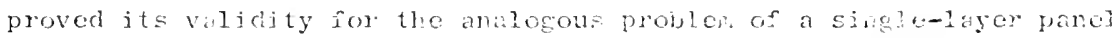
With sinilar bonidory conditions. 
When comparing the present work with existing and subsequent theories, the words panel and plate may be considered interchangeable. Numerical computatjons are carried out by a desls calculater. 
BASIC CONCEPTS

\section{Description of Sandwich Panel}

An element of the sandivich panel to be considered in this work is shoun in Figure 1. For convenience, three coordinate systems are defined having common $x$ - and $y$-cootinates, and transverse cocrolinates related as follows:

$$
\begin{aligned}
& z^{\prime}=z-\frac{t_{c}+t_{f}}{2} \\
& z^{\prime \prime}=z+\frac{t_{c}+t_{f}}{2}
\end{aligned}
$$

where $t_{c}$ is the thickness of the core and $t_{f}$ is the thickness of the face layers wich are identical. In the notational system employed here, a single prime denotes quantities related to the lower race, a double prime those related to the upper face, and umprimed quantities are used for the core as well as for goneral relations. Subscripts $c$ and $f$ denote quantities $r e^{3}$ ated to the core and face, respectively. The tota] thickness of the panel is assuned to be very small in comparison with the lateral dinensions. Also, each layer is considered to he isotropic and hrmogencous, with the pioperties of the faces ciaracterized $b_{5} E_{f}$ and $V_{f}$, and those of the core characterized by $G_{C}$. 


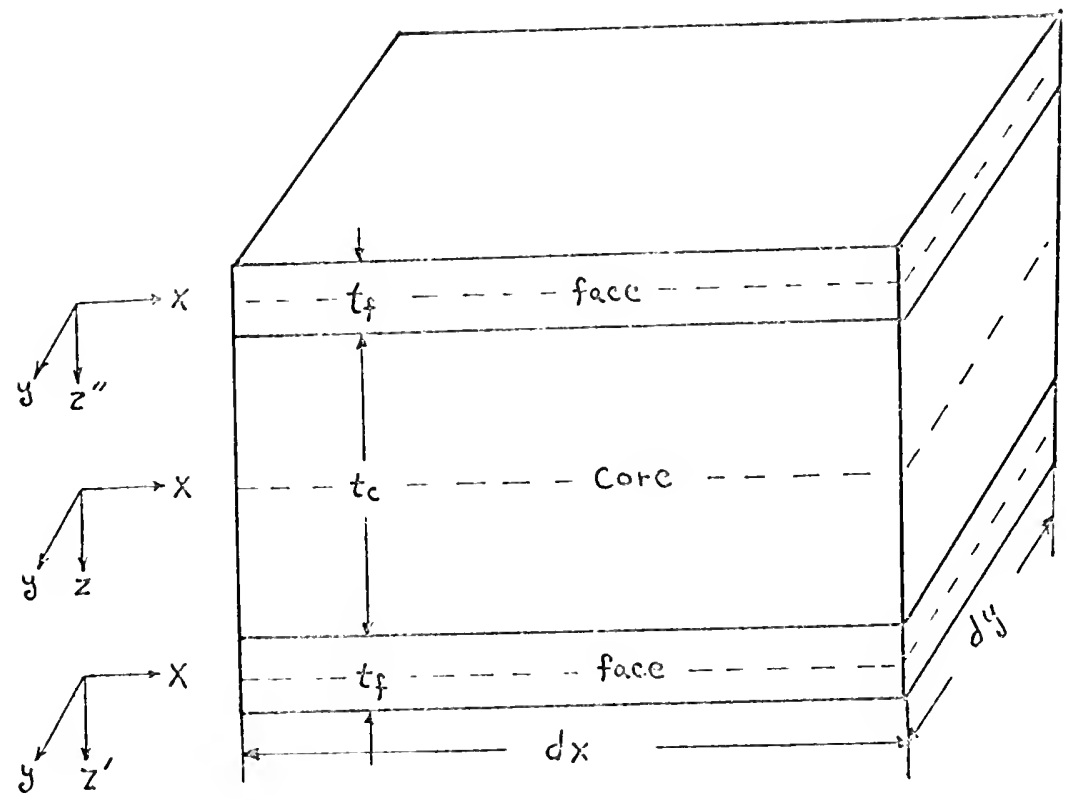

Figure 1. Element of Sandwich Fancl 
Arbjtrary transverse load $q$, axial forces $N_{-\alpha, \beta}$, and external moments $M_{-\alpha \beta}$ are the only externally applice forces or moments which may contribute work to the system.

For the initial derivation and formulations, indicial notation will be employed. Greek indices $\theta, \beta, \gamma$ and $\mu$ will take on values 1 or 2 , while Latin indices, when employed, will take on values 1,2 or 3 . Any repeaied index denotes summation, while any number of indices precedec? by a comma indicates partial differentiation with respect to the coordinate variable represented by those inclices.

\section{Displacenonts}

In the following relations, which are valicl for smali displacements, it will be assumed that within each layer of the sandwich panel plane sections are preserved, although not neccssarily perpendicular to the deflected middle surface. This will constitute an approxination since the presence of transverse shear suggests a non-linear variation of the longitudinal displacenents through the thickness. Alsc, while the panel is geometrically symetric with respect to the midcle surface, the displacenent relations will not, in general, reflect this symetry, since uncrual bending moments may be extcrnally applied to each face layer. Therefore, with the aid of equation (1), our displacements can be written in the following form:

$$
\begin{array}{ll}
\mathrm{V}_{\alpha}^{\prime}\left(\mathrm{x}, \mathrm{y}, \mathrm{z}^{\prime}\right)=u_{\alpha}^{\prime}(\mathrm{x}, \mathrm{y})+\mathrm{z}^{\prime} \dot{\psi}_{\alpha y}^{\prime}(\mathrm{x}, \mathrm{y}) & \mathrm{V}_{3}^{\prime}\left(\mathrm{x}, \mathrm{y}, \mathrm{z}^{\prime}\right)=w(\mathrm{x}, \mathrm{y}) \\
\mathrm{V}_{\alpha}(\mathrm{x}, \mathrm{y}, \mathrm{z})=z \dot{\psi}_{\alpha}(\mathrm{x}, \mathrm{y}) & \mathrm{V}_{3}(\mathrm{x}, \mathrm{y}, \mathrm{z})=w(\mathrm{x}, \mathrm{y}) \\
\mathrm{V}_{\alpha}^{\prime \prime}\left(\mathrm{x}, \mathrm{y}, \mathrm{z}^{\prime \prime}\right)=\mathrm{u}_{\alpha}^{\prime \prime}(\mathrm{x}, \mathrm{y})+z^{\prime \prime}{ }_{\alpha}^{\prime \prime}(\mathrm{x}, \mathrm{y}) & \mathrm{V}_{3}^{\prime \prime}\left(\mathrm{x}, \mathrm{y}, \mathrm{z}^{\prime \prime}\right)=w(\mathrm{x}, \mathrm{y})
\end{array}
$$


where $\left(V_{Q}, V_{3}\right)$ is the displacement of a generic point in the sandwich panel; $u_{\alpha}$ represents the displacement in the xy-plane of a point iying in the median plane of either face; represents the angle that the nomal to the nedian plane of each layer rotates when the planes are deflected; and $w$ is the transverse deflection which is assumed to be constant through the entire thichness.

In order to ensure continuily at the interface of any two adjacent layers, the following conditions must be imposed on equation (2):

$$
\begin{aligned}
& u_{c x}^{\prime}-\frac{\mathrm{t}_{f}}{2} \psi_{a}^{\prime}=\frac{{ }_{\mathrm{c}}}{2} \psi_{\alpha} \\
& u_{a}^{\prime \prime}+\frac{\mathrm{t}_{f}}{2}{ }_{a}^{\prime \prime}=-\frac{\mathrm{t}_{c}}{2} \psi_{\alpha}
\end{aligned}
$$

$$
\text { Usine (3) to elininate " and u } u_{y}^{\prime \prime} \text { from (2), our displacemert }
$$

relations becoine:

$$
\begin{array}{ll}
v_{\alpha}^{\prime}=u_{u}^{\prime}+z_{a}^{\prime} & v_{3}^{\prime}=w \\
v_{\alpha}=\frac{z}{t_{c}}\left(2 u_{Q}^{\prime}-t_{f} \psi_{G}^{\prime}\right) & v_{3}=w \\
v_{\alpha}^{\prime \prime}=-u_{Q}^{\prime}+z^{\prime \prime} \psi_{\alpha}^{\prime \prime}+\frac{t_{f}}{2}\left(\psi_{Q}^{\prime}-\psi_{Q}^{\prime \prime}\right) & v_{3}^{\prime \prime}=w
\end{array}
$$

3. Strain-Disurncencht Relations

Linear strain-displacement relations are defined by [11]:

$$
\begin{aligned}
& e_{Q \beta}=\frac{1}{2}\left(V_{Q, \beta}+v_{\beta, \alpha}\right) \\
& e_{\alpha 3}=\frac{1}{2}\left(V_{\alpha, 3}+v_{3, \alpha}\right) \\
& e_{33}=v_{3,3}
\end{aligned}
$$


Using (4) in conjunction with (5), the strain-displacement relations for each layer become:

$$
\begin{aligned}
& \mathrm{e}_{\alpha \beta}^{\prime}=\frac{1}{2}\left[u_{a, \beta}^{\prime}+z^{\prime} \psi_{O, \beta}^{\prime}+u_{\beta, \alpha}^{\prime}+z^{\prime} \psi_{\beta, \alpha}^{\prime}\right] \\
& e_{u 3}^{\prime}=\frac{1}{2}\left[\psi_{b}^{\prime}+w, q\right] \\
& \mathrm{e}_{33}^{\prime}=0 \\
& c_{\alpha \beta}=\frac{z}{2 t_{c}}\left[?_{G, \beta}^{\prime}-t_{f}^{\prime} \psi_{\alpha, \beta}^{\prime}+2 u_{\beta, Q}^{\prime}-t_{f}^{\prime} \psi_{\beta, Q x}^{\prime}\right] \\
& c_{a 3}=\frac{1}{t_{c}}\left[u_{u}^{\prime}+\frac{1}{2}\left(t_{c} w, a-t_{f}^{\prime} t_{\alpha}^{\prime}\right)\right] \\
& e_{33}=0 \\
& e_{\alpha \beta}^{\prime \prime}=\frac{1}{2}\left[u_{\alpha, \beta}^{\prime}+\frac{t_{f}}{2}\left(\psi_{\alpha, \beta}^{\prime}-\psi_{\alpha, \beta}^{\prime \prime}\right)+z^{\prime \prime} \psi_{\alpha, \beta}^{\prime \prime}\right. \\
& -u_{\beta, \alpha}^{\prime}+\frac{{ }^{t}}{2}\left(\psi_{\beta, \alpha}^{\prime}-\psi_{\beta}^{\prime \prime}, \alpha\right)+z^{\prime \prime} \psi^{\prime \prime} \beta, a^{]} \\
& e_{\alpha 3}^{\prime \prime}=\frac{1}{2}\left[\omega_{\alpha}^{\prime \prime}+w, \alpha\right] \\
& \mathrm{e}_{33}^{\prime \prime}=0
\end{aligned}
$$

4. Stress-Strain Rejations

The generalized Hocke's law for a homogeneous isotropic body can be written in the following form [15]:

$$
\tau_{i j}=\frac{\xi}{I-\gamma}\left[c_{i j}+\frac{\psi}{1-2 \nu} \delta_{i j} e_{k k}\right]
$$

where the Iatin indices $i, j$ and $b$ talce en the values 1,2 and 3 . 
Wher the transverse normal stress, $\tau_{33}$, is neglected, (7) may be rewitten as [15]:

$$
\begin{aligned}
& \tau_{\alpha \beta}=\frac{E}{1-\nu}\left[e_{\alpha \beta \beta}+\frac{\nu}{1-\nu} \delta_{\epsilon_{\beta}} e_{\gamma \gamma}\right] \\
& \tau_{\alpha 3}=\tau_{3 \alpha}=2 G c_{\alpha 3}=\frac{E}{1+\nu} c_{\alpha 3} \\
& \tau_{33}=0
\end{aligned}
$$

or,

$$
\begin{aligned}
& \tau_{\alpha 3}=\frac{E}{1-v^{2}} A_{C \beta H} \\
& \tau_{C 3}=\frac{E}{1+\nu} e_{03}
\end{aligned}
$$

where,

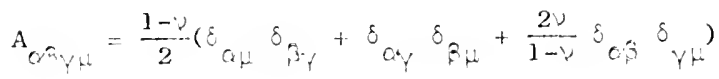

with,

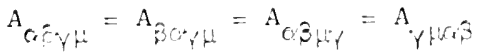

$$
\begin{aligned}
& \text { If the lateral, in-plane stresses, ' } \alpha \hat{\beta} \text { ' are also incglected, }
\end{aligned}
$$

(9) reduces to:

$$
T_{03}=\frac{E}{1+e_{03}}=2 G_{e} e_{3}
$$

Assumption (Al) implies that equations (9) may be used for the face layers, while assunption (A2) suggests the use of equation (19) for the core. 


\section{Slress Resultants}

In order to reduce the three-dimensional elasticity problem to a two-dincnsional one, the following stress resultants are defined:

$$
\begin{aligned}
& N_{\alpha \beta}^{\prime} \equiv \int_{t_{f}}^{\frac{t_{f}}{2}} \tau_{\alpha \beta}^{\prime} \mathrm{d} z^{\prime} ; N_{\alpha \beta}^{\prime \prime} \equiv \int_{t_{f \beta}}^{\frac{t_{f}}{2}} \tau_{\alpha \beta}^{\prime \prime} \mathrm{d} z^{\prime \prime} ; \\
& \begin{array}{c}
-\frac{{ }^{t}}{2} \\
-\frac{{ }^{t} f}{2}
\end{array} \\
& M_{\alpha \beta}^{\prime} \equiv \int_{t}^{\frac{t_{f}}{2}} \tau_{a \beta}^{\prime} z^{\prime} \mathrm{d} z^{\prime} ; M_{\alpha \beta}^{\prime \prime} \equiv \int_{\frac{f}{2}}^{\frac{t_{f}}{\prime \prime}} \tau_{\alpha \beta} z^{\prime \prime} \mathrm{d} z^{\prime \prime} ; \\
& -\frac{t_{f}}{2}-\frac{t_{f}}{2}
\end{aligned}
$$

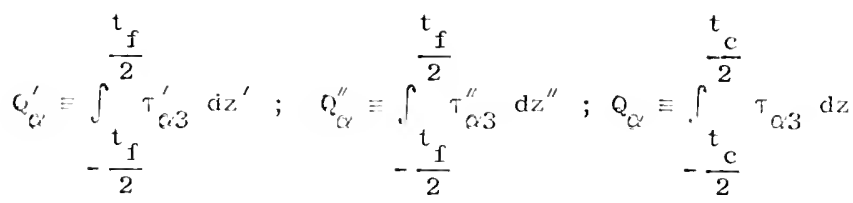

where $M, x$, and $Q$ denote internal monents, in-plane stress resultants, and shear resultants, respectively.

Expressions for stress resultants in terms of displacements are obtained by substituting strain-displacencnt relations (6) into equations (9) and (10), yielding relations for the faces and core, respectively. When these relations are then substituted into equations (13), and the indicated integration performed, the following expressions for stress, resultants and moments are obtajned: 


$$
\begin{aligned}
& \mathrm{i}_{\alpha \beta}^{\prime}=\mathrm{D}_{\mathrm{f}} \mathrm{A}_{\alpha \beta \gamma \mu} \psi_{\gamma, \mu}^{\prime} \\
& M_{\alpha \beta}^{\prime \prime}=D_{f} A_{\alpha \beta \gamma \mu} \psi_{\psi, \mu}^{\prime \prime} \\
& N_{\alpha \beta}^{\prime}=\frac{t_{f} E_{f}}{1-\nu_{f}^{2}} A_{\epsilon \beta \gamma \mu} u_{\gamma, \mu}^{\prime} \\
& N_{a \beta}^{\prime \prime}=\frac{t_{f} E_{f}}{1-\nu_{f}^{2}} A_{\alpha \beta, \mu}\left[-u_{\gamma, \mu}^{\prime}+\frac{t_{f}}{2}\left(\psi_{\gamma, \mu}^{\prime}-\psi_{\gamma, \mu}^{\prime \prime}\right)\right] \\
& Q_{\alpha}^{\prime}=\frac{\mathrm{t}_{f} \mathrm{E}_{\mathrm{f}}}{2\left(1+L_{f}\right)}\left[\dot{\gamma}_{\alpha}^{\prime}+\mathrm{w}, \alpha\right] \\
& Q_{\alpha}^{\prime \prime}=\frac{t_{f} E^{\prime}}{2\left(1+L_{f}\right)}\left[t_{\alpha}^{\prime \prime}+w_{,}\right] \\
& Q_{G}=2 G_{C}\left[u_{\sigma}^{\prime}+\frac{1}{2}\left(1 c_{c}{ }^{\prime}, a^{\prime}-t_{f} \psi_{\alpha}^{\prime}\right)\right]
\end{aligned}
$$

where the bending rigidity of the faces, ${ }_{f}$, is defined:

$$
D_{f} \frac{E_{f} t_{f}^{3}}{12\left(1-v_{f}^{2}\right)}
$$




\section{CHAPTLR I I I}

\section{DERIVATION OF EQUATIONS IN CARTESIAN COORDINATES}

\section{Total Potential}

The total potential consists of the strain energy stored in the panel during a small deformation and the total work performed on the panel by the external forces and moments during that deformation. In general, the strajn energy stored in the body is defineit [14]:

$$
\mathrm{U}=\frac{1}{2} \iint_{\mathrm{V}} \int_{i j} \tau_{i j} \mathrm{dV}
$$

In particular, as a result of assumptions (A1) and (A2), the strain energies associated with the faces and core are:

$$
\begin{aligned}
& \mathrm{U}_{f}^{\prime}=\frac{1}{2} \iint_{\mathrm{v}} \int_{\alpha 3}\left(\tau_{\alpha \beta}^{\prime} \mathrm{e}_{\alpha \beta}^{\prime}+2 \tau_{\alpha 3}^{\prime} \mathrm{e}_{\alpha 3}^{\prime}\right) \mathrm{dv}^{\prime} \\
& \left.\mathrm{v}_{\mathrm{c}}=\frac{\mathrm{J}}{2} \cdot \int_{\mathrm{v}}^{0} \int_{\alpha 3} \mathrm{e}_{u 3}\right) \mathrm{dv} \\
& \mathrm{U}_{f}^{\prime \prime}=\frac{1}{2} \iiint_{\mathrm{v}}^{\prime \prime}\left(\tau_{\alpha \beta}^{\prime \prime} \mathrm{e}_{\alpha \beta}^{\prime \prime}+2 \tau_{\alpha 3}^{\prime \prime} \mathrm{e}_{\alpha 3}^{\prime \prime}\right) \mathrm{dv} \mathrm{v}^{\prime \prime}
\end{aligned}
$$

where the integration is performed over the volumes of the incluiciual layers. 
Also, since the only externally applied forces or moments which are allowed to do work are the transverse loading function, $q$, the axial forces $\underline{N}_{-\alpha}$, and the external moments ${ }_{-\alpha}$, , the worl: performed on the panel is defined by:

$$
\begin{aligned}
& W_{\mathrm{q}}=\int_{\mathrm{a}} \mathrm{q} \mathrm{w} \mathrm{da} \\
& W_{N}^{\prime}=-\frac{1}{4} \prod_{a} N_{\alpha \beta}\left(v_{\alpha, \beta}^{\prime}+v_{B, \alpha}^{\prime}+w_{, \alpha}{ }^{w}, \beta\right) \mathrm{da}^{\prime} \\
& w_{X}^{\prime \prime}=-\frac{1}{4} \iint_{a} N_{\alpha \beta}\left(v_{\alpha, \beta}^{\prime \prime}+v_{3, \alpha}^{\prime \prime}+w_{, \alpha}{ }^{w}, \beta\right) \mathrm{da}^{\prime \prime} \\
& \left.W_{M}=\int_{S} \underline{M}_{-\alpha \beta}^{\prime} \psi_{\alpha}^{\prime}+\underline{M}_{-\alpha \beta}^{\prime \prime} \psi_{\alpha}^{\prime \prime}\right) \mathrm{n}_{\beta} \mathrm{ds}
\end{aligned}
$$

where the integration is performed over the areas and bourdaries of the individual 1 ayere.

In the secomel and third integral expressions of (15), which are analogotis to those employed by Timoshento [3] and Eringen [5], it is assumed tirst the total axjal load is evenly distributed betreen the unper and lower faces.

Therefore, the total potential, which is defined as

$$
v=U_{f}^{\prime}+U_{c}+U_{f}^{\prime \prime}-W_{q_{1}}-W_{N}^{\prime}-w_{N}^{\prime \prime}-W_{N 1}
$$

may be rowitten, using (14) and (15), in the following form: 


$$
\begin{aligned}
& v=\frac{1}{2} \int_{v} \int_{v^{\prime}}\left(\tau_{\alpha}^{\prime} e_{\alpha \beta}^{\prime}+2 \tau_{\alpha 3}^{\prime} e_{\alpha 3}^{\prime}\right) d v^{\prime} \\
& +\frac{1}{2} \iint_{v} \int_{\alpha 3}\left(2 \tau, e_{\alpha}\right) d v+\frac{1}{2} \iint_{v}^{\prime \prime}\left(\int_{\alpha \beta}^{\prime \prime} c_{\alpha \beta}^{\prime \prime}+2 \tau_{\alpha 3}^{\prime \prime} e_{\alpha 3}^{\prime \prime}\right) d v^{\prime \prime} \\
& -\int_{a} \mathrm{q} w \mathrm{da}+\frac{1}{4} \int_{a}^{\beta} \mathrm{N}_{\alpha \beta}\left(\mathrm{V}_{\alpha, \beta}^{\prime}+\mathrm{v}_{\alpha, \alpha}^{\prime}+w^{\prime}, \alpha{ }^{\prime}, \beta\right) \mathrm{da}^{\prime} \\
& +\frac{1}{4} \iint_{a} N_{\alpha \beta}\left(v_{\alpha, \beta}^{\prime \prime}+v_{\beta, \alpha}^{\prime \prime}+w, \alpha w_{, \beta}\right) \text { da" } \\
& \text { - } \left.\int_{S}{ }_{-\alpha \beta} \psi_{\alpha}^{\prime}+\frac{M^{\prime \prime}}{-a \beta} \psi_{\alpha}^{\prime \prime}\right) n_{3} d s
\end{aligned}
$$

\section{Theorem of nininum Potentjal Energy}

The theorm of minimm potential energy states that of all displacements satjsfying the givon bommary conditions, those wich satisfy the cquiljbrim equations make the total potential crergy an absolute minimum [14]. Therefore, eruilibrium equations and boundary conditions for the santwich panel are given by the variational equation:

$$
\delta v=0
$$

Howerer, before applying this extresun principle to equation (17), vie note that:

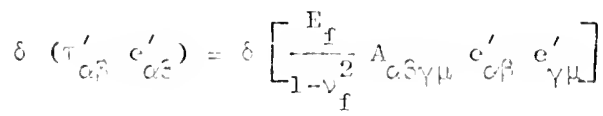

$$
\begin{aligned}
& =\frac{1}{1-\nu_{1}^{2}} A_{\alpha L_{Y H}} \epsilon_{\alpha \beta}^{\prime} \delta \Theta^{\prime}+\mu \\
& =2 i_{0}^{\prime} \& e_{0}^{\prime}
\end{aligned}
$$


and similarly,

$$
\begin{aligned}
& \delta\left(\tau_{\alpha 3}^{\prime} e_{\alpha 3}^{\prime}\right)=2 \tau_{\alpha 3}^{\prime} \delta e_{\alpha 3}^{\prime} \\
& \delta\left(\tau_{\alpha 3} e_{\alpha 3}\right)=2 \tau_{\alpha 3}{ }_{\alpha} e_{\alpha 3} \\
& \delta\left(T_{\alpha 3}^{\prime \prime} e_{\alpha 3}^{\prime \prime}\right)=2 \tau_{\alpha 3}^{\prime \prime} \delta e_{\alpha 3}^{\prime \prime} \\
& \delta\left(\tau_{\alpha \beta}^{\prime \prime} e_{\alpha \beta}^{\prime \prime}\right)=2 \tau_{\alpha \beta}^{\prime \prime} \delta e_{\alpha}^{\prime \prime}
\end{aligned}
$$

Therefore, using cquations (19-23) in conjunction with displacement relations ( 1 ) and strain-displacment relations (6), the valiation of equation (17) yields:

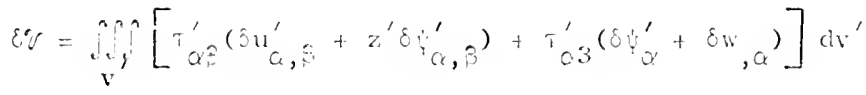

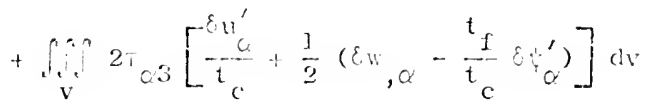

$$
\begin{aligned}
& +\int_{v^{\prime \prime}}\left\{\tau_{0, \beta}^{\prime \prime}\left[-\delta_{\alpha, \beta}^{\prime}+z^{\prime \prime} \delta \gamma_{\alpha, \beta}^{\prime \prime}+\frac{t}{2}\left(\delta \psi_{\alpha, \beta}^{\prime}-\delta \psi_{\alpha, \beta}^{\prime \prime}\right)\right]\right. \\
& \left.+\tau_{\alpha 3}^{\prime \prime}\left[\delta \psi^{\prime \prime}+\delta w, \alpha\right]\right\} d \mathrm{v}^{\prime \prime} \\
& -\iint_{a} q \delta w c i a+\frac{1}{2} \iint_{a} N-a,\left[2 w, \alpha w, \beta+\frac{t}{2}\left(\delta \psi_{\alpha, \beta}^{\prime}-\delta v_{\alpha, \beta}^{\prime \prime}\right)\right] d a \\
& -\int_{S}\left[\frac{i^{\prime}}{-a_{2}} \delta t_{\alpha}^{\prime}+M_{\alpha}^{\prime \prime} \delta \psi_{\alpha}^{\prime \prime}\right] n_{\hat{S}} d s=0
\end{aligned}
$$

In equation (21) the symetry of the stress tensors and strain tensors has heen employer. Also, the djoplacoment: 
associated witli Nos are those occurring in the median planes of the face 1 aycrs.

Using relations (11) to reduce the volume integrals in equation (2:), and applying the two-dimensional divergence theorer. of Gauss to the resulting area integrals, the final integral ecuation is obtained.

As an example of the above procedure, coisider the fiost torit in the integrand of equation $(2,1)$ :

$$
\begin{aligned}
& \iiint_{\alpha}^{\prime} \tau_{\alpha}^{\prime} \delta u_{\alpha, \beta}^{\prime} d v^{\prime}=\iint_{a}^{t} \int_{-t / 2}^{t / 2} \tau_{\alpha \beta}^{\prime} \delta u_{\alpha, \beta}^{\prime} d z^{\prime} d a \\
& =\int_{a} N_{\alpha \beta}^{\prime} \delta u_{\alpha, \beta}^{\prime} d a \\
& \left.=\int_{a}\left[{ }_{\alpha \beta}{ }^{\prime \prime} u_{\alpha}^{\prime}\right), \beta-N_{\alpha \beta, \beta}^{\prime} \delta u_{\alpha}^{\prime}\right] \mathrm{da} \\
& =\int_{s} x_{\alpha \beta}^{\prime} \delta u_{\alpha}^{\prime} n_{\beta} \text { ds }-\iint_{a} x_{\alpha \beta}^{\prime}, \delta u_{\alpha}^{\prime} \text { da }
\end{aligned}
$$

After applying a similur procedure to the remaining terms, and collecting coefficients of similar virtua displacenents, a conplete description of the sandwich pancl in the equilibriun state is obtajned in interral ior:l:

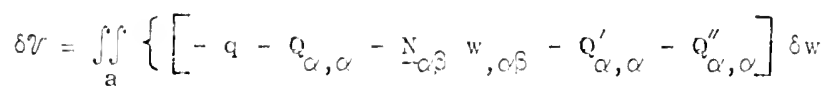

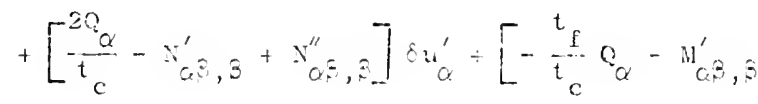




$$
\begin{aligned}
& \left.\left.+Q_{\alpha}^{\prime}-\frac{t_{f}}{2} N_{\alpha \beta, \beta}^{\prime \prime}\right] \delta \psi_{\alpha}^{\prime}+\left[\frac{f}{2} N_{\alpha \beta, \beta}^{\prime \prime}-M_{\alpha \beta, \beta}^{\prime \prime}+Q_{\alpha}^{\prime \prime}\right] \delta \psi_{\alpha}^{\prime \prime}\right\} d i \\
& +\int_{S}\left\{\left[Q_{\alpha}+N_{\alpha \beta} w, \beta+Q_{\alpha}^{\prime}+Q^{\prime \prime}\right] \delta w\right.
\end{aligned}
$$

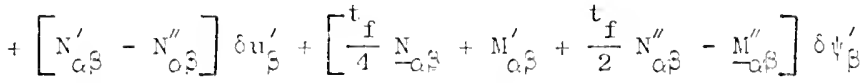

$$
\begin{aligned}
& \left.+\left[-\frac{t_{f}}{4} N_{-\alpha \beta}-\frac{t_{f}}{2} N_{\alpha \beta}^{\prime \prime}+N_{\alpha \beta}^{\prime \prime}-N_{\alpha \beta}^{\prime \prime}\right] \delta \psi_{\beta}^{\prime \prime}\right\} n_{\alpha} d s=0
\end{aligned}
$$

\section{Equilibrium Equations and}

\section{Bomantry Conditions}

If we apply the funcianental lema of the calculus of variations [15] to cquation (26), we obtain the following equilibrium equations in terms of stress resultants and moments:

$$
\begin{aligned}
& -\mathrm{N}_{-\alpha}{ }^{\mathrm{W}}, \mathrm{C}_{3}-\left(\mathrm{Q}_{\alpha}+\mathrm{O}_{\alpha}^{\prime}+\mathrm{Q}_{\alpha}^{\prime \prime}\right), a=\mathrm{q} \\
& \frac{2 Q_{\alpha}}{t_{c}}-N_{\alpha \beta, \beta}^{\prime}+N_{\alpha \beta, \beta}^{\prime \prime}=0 \\
& -\frac{t_{f}}{t_{c}} Q_{\alpha}-n_{\alpha_{j}^{\prime}, \beta}^{\prime}+Q_{\alpha}^{\prime}-\frac{t^{\prime}}{2} N_{\alpha \beta, \beta}^{\prime \prime}=0 \\
& \frac{t_{1}}{2} N_{\alpha \beta, \beta}^{\prime \prime}-N_{\alpha \beta, \beta}^{\prime \prime}+Q_{\alpha}^{\prime \prime}=0
\end{aligned}
$$

and boundary concilions,

$$
\begin{aligned}
& \int_{s}\left[Q_{\alpha}+Q_{\alpha}^{\prime}+Q_{\alpha}^{\prime \prime}+N_{0 \beta} w_{\beta}\right] \delta \mathrm{w}_{\alpha} \text { is }=0 \\
& \int_{s}\left[N_{\alpha}^{\prime}-N_{\alpha \beta}^{\prime \prime}\right] \delta u_{\alpha}^{\prime} n_{\beta} \mathrm{ds}=0
\end{aligned}
$$




$$
\begin{aligned}
& \int_{s}\left[\frac{f}{4} N_{-\alpha \beta}+M_{\alpha \beta}^{\prime}+\frac{t_{f}}{2} N_{\alpha \beta}^{\prime \prime}\right] \delta \psi_{\alpha}^{\prime} n_{\beta} d s=0 \\
& \int_{s}\left[-\frac{1}{4} \underline{N}_{\alpha \beta}+M_{\alpha \beta}^{\prime \prime}-\frac{t_{f}}{2} N_{\alpha \beta}^{\prime \prime}\right] \delta \psi_{\alpha}^{\prime \prime} n_{\beta} d s=0
\end{aligned}
$$

If we substitute the stress resultant-displacement relations (12) into equations (27) and (2S), and collect similar terms, we obtain the following equilibritu equations in terms of displacements:

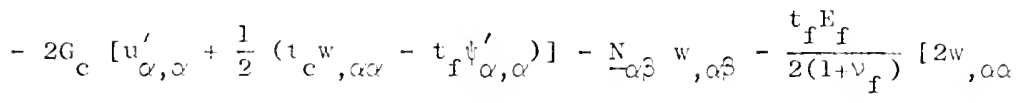

$$
\begin{aligned}
& \left.+\left(\psi_{\alpha, \alpha}^{\prime} \alpha^{+} u^{\prime \prime}, \alpha\right)\right]=q
\end{aligned}
$$

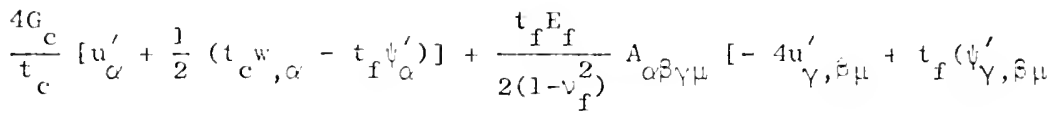

$$
\begin{aligned}
& \left.\left.-\dot{\psi}_{Y, \hat{\rho}_{\mu}}^{\prime \prime}\right)\right]=0
\end{aligned}
$$

$$
\begin{aligned}
& \frac{-2 t_{f}^{G}{ }_{c}}{t_{c}}\left[u_{\alpha}^{\prime}+\frac{1}{2}\left(t_{c} w^{\prime}, \alpha-t_{f^{\prime} \psi_{\alpha}^{\prime}}\right)\right]+\frac{t_{f} F_{f}}{2\left(1+v_{f}\right)}\left[\psi_{\alpha}^{\prime}+w, \alpha\right]
\end{aligned}
$$

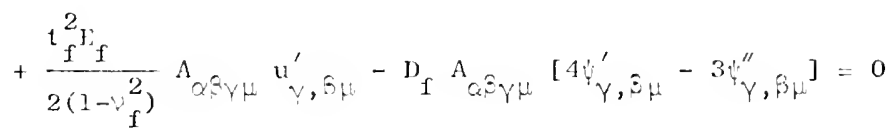

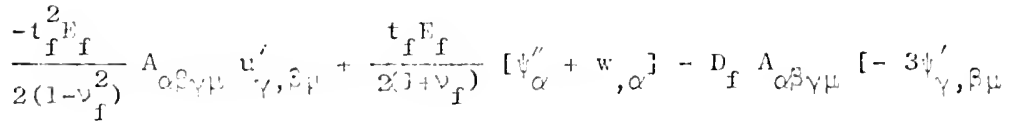

$$
\begin{aligned}
& \left.+4 \psi_{\gamma, \rho,}^{\prime \prime}\right]=0
\end{aligned}
$$


and boundary conditions,

$$
\begin{aligned}
& \int_{s}\left\{2 G_{C}\left[u_{\alpha}^{\prime}+\frac{1}{2}\left(t_{c} w, \alpha-t_{f^{\psi}} \psi_{\alpha}^{\prime}\right)\right]+N_{-\alpha \beta} w_{, \beta}+\frac{t_{f} E_{f}}{2\left(1+\nu_{f}\right)}[2 w, \alpha\right. \\
& \left.\left.+\psi_{\alpha}^{\prime}+\psi_{\alpha}^{\prime \prime}\right]\right\} w \mathrm{n}_{\alpha} \mathrm{ds}=0 \\
& \int_{s}\left\{\frac{t_{f} E_{f}}{2\left(1-v_{f}^{2}\right)} A_{\alpha}{ }^{3} \gamma \mu \mu_{\gamma, \mu}\left[1 u^{\prime}-t_{f}\left(\psi^{\prime}{ }_{\gamma, \mu}-\psi_{\gamma, \mu}^{\prime \prime}\right)\right]\right\} \varepsilon_{\alpha a_{\alpha}^{\prime}} n_{\beta} \text { ds }=0
\end{aligned}
$$

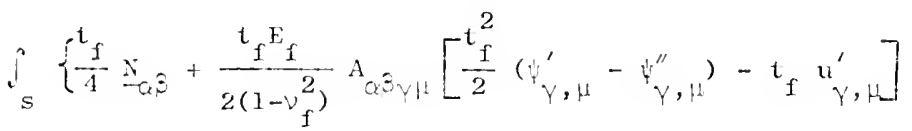

$$
\begin{aligned}
& \left.+n_{f} A_{\alpha \beta \gamma \mu} \psi_{\gamma, \mu}^{\prime}-N_{\alpha} \beta\right\} \delta \psi_{\alpha}^{\prime} n_{\beta} d s=0
\end{aligned}
$$

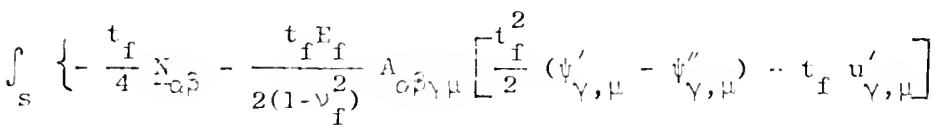

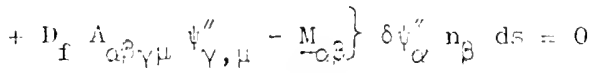

Boundary conditions (30) state that either the quantities contaired within the lapege brackets must vanish along the boundary, or the variation of the displacenent must vanish along the boundary.

$$
\text { Compared with equations (27) and (28), equations (28) and }
$$

(30) constituie a formulation through which the bucking problem can be nore readizy soived. This becomes immediately evident by considering the classical concept of instability which is associated with displacement fields rather than stress fields. 


\section{Simplification of Equations}

In order to simplify equations (29) and (30), we exclude the possibility of externally applied edge moments by specifying:

$$
M_{\alpha \beta}^{\prime}=M_{-\alpha \beta}^{\prime \prime}=0
$$

which reduces the number of dependient variables from seven to five since (31) suggests:

$$
\psi_{\alpha}^{\prime}=\psi_{\alpha}^{\prime \prime}
$$

Also, if we assume that plane sections remain perpendicular to the deflected middle sulface of each respective face (asssumption Bit), we have,

$$
\psi_{\alpha}^{\prime}=\psi_{\alpha}^{\prime \prime}=-w, \alpha
$$

Which further reduces the number of dependent variables to three.

A final assumption (132) wich simplifies the problem by redueing the order of the differential equations is [1]:

$$
\mathrm{t}_{\mathrm{c}} \gg \mathrm{t}_{\mathrm{f}}
$$

Which justifics nerlecting the bending rigidity of the facos, $\mathrm{D}_{\mathrm{f}}$, compared with the bending rigidity of the panel as a whole.

Consecuently: when equations (29) and (30) are simplificd through the apjlication of $(31-31)$, we obtain the following entilibrium equations: 


$$
\begin{aligned}
& -\hat{t} t_{c} G_{c}\left(\frac{2}{t_{c}} u_{\alpha, \alpha}+\hat{t} w, \alpha \alpha\right)-N_{\alpha \beta}{ }^{w}, \alpha \beta=q \\
& 2 G_{c}\left[\frac{2}{t_{c}} u_{\alpha}+\hat{t} w, \alpha\right]-\frac{2 t_{f} E_{1}}{1-\nu_{f}^{2}} A_{\alpha \beta \gamma \mu}{ }^{u}{ }_{\gamma}, \beta \mu=0
\end{aligned}
$$

and boundary conditions,

$$
\begin{aligned}
& \int_{s}\left\{2 \hat{t} t_{c} G_{c}\left[\frac{\alpha}{t_{c}}+\frac{\hat{t}}{2} w, \alpha\right]+N_{-\alpha \beta} w, \beta\right\} \varepsilon_{w} n_{\alpha} d s=0 \\
& \int_{s}\left\{\frac{f_{1}^{E_{f}}}{2} A_{\alpha \beta_{\gamma} \mu} u_{\gamma, \mu}\right\} \varepsilon_{\alpha} u_{\alpha} \mathrm{n}_{\beta} \mathrm{ds}=0
\end{aligned}
$$

Equations (35) are (36) can be rewritten in extended form as:

$$
\begin{aligned}
& -\hat{\imath} t_{c} G_{c}\left[\frac{2}{t} u_{c}, x+\hat{\imath} \psi, x y-\hat{\imath} t_{c} G_{c}\left[\frac{2}{t_{c} u_{y}, y}+\hat{\imath} w, y\right]\right]
\end{aligned}
$$

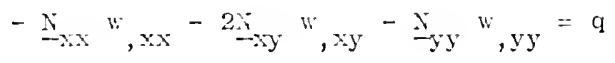

$$
\begin{array}{r}
2 G_{C}\left[\frac{2}{t_{c}} u_{x}+\hat{\imath} u, x\right]-\frac{t_{f} E_{f}}{1-v_{f}^{2}}\left[\left(1+v_{f}\right)\left(u_{x, x x}+u_{y, x y}\right)\right. \\
\left.+\left(1-v_{f}\right)\left(u_{x, x x}+u_{x, y y}\right)\right]=0
\end{array}
$$

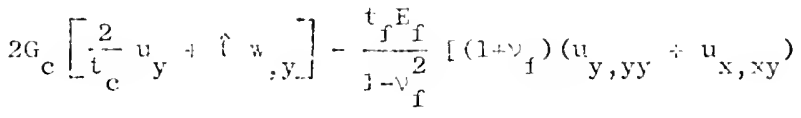

$$
\begin{aligned}
& +\left(1-\nu_{f}\right)\left(u_{y, x x}+u_{y, y, y}\right]=0
\end{aligned}
$$


and,

$$
\begin{aligned}
& \int^{y}\left\{2 \hat{t} t_{c} G_{c}\left[\frac{\mathrm{u}}{\mathrm{t}}+\frac{\hat{\mathrm{t}}}{2} w, x\right]+\mathrm{N}_{\mathrm{x} x}{ }^{\mathrm{w}}, \mathrm{x}+\mathrm{N}_{\mathrm{xy}}{ }^{\mathrm{w}}, \mathrm{y}\right\} \delta \mathrm{w} \mathrm{n}_{\mathrm{x}} \mathrm{dy}=0
\end{aligned}
$$

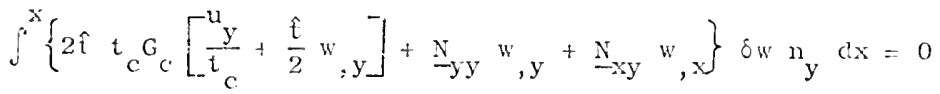

$$
\begin{aligned}
& \bigcup^{y}\left\{\frac{2 t_{f} E_{f}}{1-\nu_{f}^{2}}\left[u_{x, x}+\nu_{f} u_{y, y}\right]\right\} \delta u_{x}{ }_{x} \text { dy }=0 \\
& \int\left\{\frac{f^{E} f}{1+v_{f}}\left[u_{x, y}+u_{y, x}\right]\right\} \delta u_{y} n_{x} d y=0 \\
& \int^{x}\left\{\frac{2 f^{F} f}{1-v_{f}^{2}}\left[u_{y, y}+v_{f} u_{x, x}\right]\right\} \delta u_{y} n_{y} d x=0 \\
& \int^{x}\left\{\frac{f^{E} f}{1+v_{f}}\left[u_{x, y}+u_{y, x}\right]\right\} \delta u_{x} n_{y} d x=0 \\
& \text { where } \hat{t} \equiv \frac{t_{c}+t_{f}}{t_{c}} \text {, }
\end{aligned}
$$

and primes and doublc prines have been onit ted without confusion as a vesult of equation (31).

Equations ( 37 ), which are a spccial case of equations (29), are identical with those derjued by Chang and Lbejoglu [23] jf thermoelostic effeets ame negiceted.

It shcuid be noted that when simplification (3a) is introduced into equations (29) ard (30), equations (36) and (36) are loot imediatriy produced. The last 1 wo of equations (29) nust be returned to the area integral of equation (26) wherc, as a reate of (33), they become coefficients of - E,$Q^{*}$ Concequently, a transfomation puce-

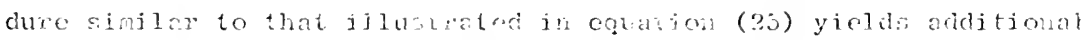
terms wict contrjbute to chutione (35) and (30). 
EQUILIDRIUN EQUATIONS AND BOUNDARY CONDITIOAS

IN CYLINDRI CAL COORDINATES

\section{Appication of Covariant Derivatives}

Let us consider a covariant clerivative of an arbitrary

covarjant vector, $x_{n},[16]$ :

$$
x_{n / q}=\frac{\partial x_{n}}{\partial x^{q}}-\left\{\begin{array}{c}
l \\
n q
\end{array} x_{l}\right.
$$

In (39) $x^{4}$ is at general coordinate variable, $\left\{\begin{array}{l}b \\ \text { nq }\end{array}\right\}$ is the Christoffel symbol of the sccond kinci, and all indices take on values 1, 2, or 3. Iurthermore, when a vertical line prececles any number of indices, it indicates covarant differentiation with respect to the cooddinate variabies represented by those indices.

In considering the second covariant derivative of an arbitraxy vector we Inust recall that in ordar to sum two indices we riust have one covariant and onc contravariant index. Therefore, introdricing the metric tensor, $\mathrm{g}^{\mathrm{nq}}$, (39) implics:

$$
x_{n \mid} s=g^{s q} x_{n \mid q}=g^{s q}\left[\frac{\partial x^{n}}{\partial x_{q}^{q}}-\left\{r_{n q}^{\ell} x_{\ell}\right]\right.
$$

And since the covariani derivative of an aribitrary second order mixed tensor, $Y_{n}^{s}$, is $\left[\begin{array}{ll}1 & 6\end{array}\right]$ : 


$$
Y_{n \mid t}^{s}=\frac{\partial Y_{n}^{s}}{\partial x^{t}}+\left\{\begin{array}{c}
s \\
m t
\end{array}\right\} Y_{n}^{n}-\left\{\begin{array}{c}
m \\
n t
\end{array}\right\} Y_{m}^{s}
$$

we have, from (40) and (41):

$$
\begin{aligned}
& x_{n \mid p t}=g_{p s} x_{n \mid t}^{s}=g_{p s}\left\{\frac { \partial } { \partial x ^ { t } } \left[g^{s q} \frac{\partial x_{n}}{\partial x^{q}}\right.\right. \\
& -g^{s q}\left\{\begin{array}{c}
\ell \\
n q
\end{array}\right\} x,+\left\{\begin{array}{c}
s \\
m t
\end{array}\right\}\left[g^{m q} \frac{\partial x}{\partial x^{q}}-g^{m q}\left\{\begin{array}{c}
\ell \\
n q
\end{array}\right\} x_{\ell}\right] \\
& -\left\{\begin{array}{c}
m \\
n t
\end{array}\right\}\left[g q^{s} \frac{\partial x}{\partial x^{q}}-g^{s q}\left\{\begin{array}{c}
k \\
m q
\end{array} x_{l}\right]\right\}
\end{aligned}
$$

In cylincirical coordinates, the Christuffel symbols and metric tensor take the following form [16]:

$$
\begin{gathered}
\left\{\begin{array}{c}
2 \\
12
\end{array}\right\}=\left\{\begin{array}{c}
2 \\
21
\end{array}\right\}=\frac{1}{x^{1}} ; \quad\left\{\begin{array}{c}
1 \\
22^{2}
\end{array}=-x^{1}\right. \\
\text { all other }\left\{\begin{array}{c}
\mathrm{n} \\
\mathrm{sin}^{3}
\end{array}\right\}=0 \\
\mathrm{~g}_{11}=\mathrm{g}_{33}=\mathrm{g}^{11}=\mathrm{g}^{33}=1 \\
\mathrm{~g}_{22}=\left(x^{1}\right)^{2} ; \quad \mathrm{g}^{22}=\frac{\left(\frac{1}{x^{1}}\right)^{2}}{x^{2}}
\end{gathered}
$$

Equations (42) and (43) imply:

$$
x_{n \mid p t}=-x_{n} \mid t p
$$

Therefore, with the aid of (13) and (44), (39) and (42) may be expanded to yield: 


$$
\begin{aligned}
& x_{1 / 1}=\frac{\partial x_{1}}{\partial x^{1}} \\
& x_{2 / 2}=\frac{\partial x_{2}}{\partial x^{2}}+x^{1} x_{1} \\
& x_{1 / 2}=\frac{\partial x_{1}}{\partial x^{2}}-\frac{1}{x^{1}} x_{2} \\
& x_{2 / 1}=\frac{\partial x_{2}}{\partial x^{1}}-\frac{1}{x^{1}} x_{2} \\
& x_{3 / 2}=\frac{\partial x_{3}}{\partial x^{2}} \quad x_{3 / 1}=\frac{\partial x_{3}}{\partial x^{1}}
\end{aligned}
$$

and,

$$
\begin{aligned}
& x_{1 \mid 11}=\frac{\partial^{2} x_{1}}{\partial\left(x_{0}^{1}\right)^{2}} \\
& x_{1 \mid 22}=\frac{\partial^{2} x_{1}}{\partial\left(x^{2}\right)^{2}}-\frac{2}{x^{1}} \frac{\partial x_{2}}{\partial x^{2}}+x^{1} \frac{\partial x_{1}}{\partial x^{1}}-x_{1} \\
& x_{1 \mid 12}=\frac{\partial^{2} x_{1}}{\partial x^{1} \partial x^{2}}-\frac{1}{x^{1}} \frac{\partial x_{1}}{\partial x^{2}}+\frac{2 x_{2}}{\left(x^{1}\right)^{2}}-\frac{1}{x^{1}} \frac{\partial x_{2}}{\partial x_{1}^{1}} \\
& x_{2 \mid 11}=\frac{\partial^{2} x_{2}}{\partial\left(x^{1}\right)^{2}}+\frac{2 x_{2}}{\left(x^{1}\right)^{2}}-\frac{2}{x^{1}} \frac{\partial x_{2}}{\partial x^{1}} \\
& x_{2 \mid 22}=\frac{\partial^{2} x_{2}}{\partial\left(x^{2}\right)^{2}}+2 x^{1} \frac{\partial x_{1}}{\partial x^{2}}+x^{1} \frac{\partial x_{2}}{\partial x^{1}}-2 x_{2} \\
& x_{2 \mid 12}=\frac{\partial^{2} x_{2}}{\partial x^{1} x^{2}}-\frac{2}{x^{1}} \frac{\partial x_{2}}{\partial x^{2}}+x^{1} \frac{\partial x_{1}}{\partial x^{1}}-x_{1}
\end{aligned}
$$




$$
\begin{aligned}
& x_{3 / 11}=\frac{\partial^{2} x_{3}}{\partial\left(x^{1}\right)^{2}} \\
& x_{3 / 12}=\frac{\partial^{2} x_{3}}{\partial x^{1} \partial x^{2}}-\frac{1}{x^{1}} \frac{\partial x_{3}}{\partial x^{2}} \\
& x_{3 / 22}=\frac{\partial^{2} x_{3}}{\partial\left(x^{2}\right)^{2}}+x^{1} \frac{\partial x_{3}}{\partial x^{1}}
\end{aligned}
$$

In order to make each equation of (45) and (46) dimensionally homogcneous, we replace the right-hand members by thejr physical components, $\mathrm{x}_{(\mathrm{n})}$, through the relatjonship [16]:

$$
x_{(n)}=\sqrt{g^{n n}} x_{n} \quad \text { (No sumation) }
$$

where a biaclieted indes inclicates plysical component.

Furthernore, to cmsure dimensional comatibility among equations, the following relations are used to introduce physical compononts to the loft-hand nembers of cquations (45) and (46):

$$
\begin{aligned}
& x_{(n) \mid(l)}=\sqrt{g^{m n}} \sqrt{g l l} x_{n \mid l} \\
& x_{(n) \mid(l)(p)}=\sqrt{g^{n n}} \sqrt{g^{l \cdot l}} \sqrt{g^{p p}} x_{n \mid l p} \quad \text { (Ne sumnation) }
\end{aligned}
$$

The lcft-hand mamber's of the resulting cquations nay be interpreted as cartesjan componchts. Therefore, after renacing $x^{1}$ by $r, x^{2}$ by $\theta, x_{(i)}$ by $u_{r}, x_{(2)}$ by $u_{\theta}$, and $x_{(3)}$ by w in the $r i g h t-$ hairl menters, and $x_{(1)}$ by $u, x_{(2)}$ by $u_{y}$, and $x_{(3)}$ by $w$ in the lesthand nembers, trensfometion cquatjons relating cartesian and polar coordinates talic the following furm: 


$$
\begin{aligned}
& u_{x}=u_{r} \\
& u_{y}=u_{\theta} \\
& u_{x / x}=u_{r, r} \\
& \left.u_{y}\right|_{y}=\frac{1}{r} u_{\theta, \theta}+\frac{1}{r} u_{r} \\
& \left.u_{x}\right|_{y}=\frac{1}{r} u_{r, e}-\frac{1}{r} u_{\theta} \\
& u_{1}{ }_{x}={ }^{\mathrm{e}}, \mathrm{x} \\
& n=w, r \\
& W y=\frac{1}{y} w, \theta \\
& u_{x \mid x x}={ }^{u} r, r x \\
& u_{x, y y}=\frac{1}{r^{2}}{ }^{u_{r}} r, \theta \theta-\frac{2}{r^{2}} u_{\theta, \theta}+\frac{1}{r} u_{r, r}-\frac{1}{r^{2}} u_{r} \\
& \left.u_{x}\right|_{x y}=\frac{1}{3} u_{r, r}-\frac{1}{r^{2}} u_{r, \theta}-\frac{1}{r} u_{\theta, r}+\frac{1}{r^{2}} u_{\theta} \\
& u_{y \mid x x}=u_{\theta, x r} \\
& { }_{y / y}=\frac{1}{r} u_{\theta, \theta \theta}+\frac{2}{r^{2}} u_{r, \theta}+\frac{1}{r} u_{\theta, r}-\frac{1}{r^{2}} u_{\theta} \\
& u_{y} \mid x y_{y}=\frac{1}{r} u_{\theta, r} \theta-\frac{1}{x^{2}} u_{\theta, \theta}+\frac{1}{1} u_{r, r}-\frac{1}{2} u_{r} \\
& w / x:=w, r r \\
& \eta_{y y}=\frac{1}{r} w, r \theta-\frac{1}{r^{2}} \because, \hat{\theta} \\
& \forall y=\frac{1}{r^{2}} w, \theta \theta \div \frac{1}{r} w, r^{2}
\end{aligned}
$$


In equation (49), $r$ and $\theta$ are polar coordinates defined in the usual manner, while ${ }_{r}$, " ${ }_{\theta}$ and w represent displacements in the directions associated with this new coordinate system.

\section{Transformatjon of Equations}

If we intorpret the derivatives appearing in equations (37) and $(3 S)$ as covariant derivatives and thereby transforn then into polar cooritrates using relations (49), we arrive at the following equilibrium equations:

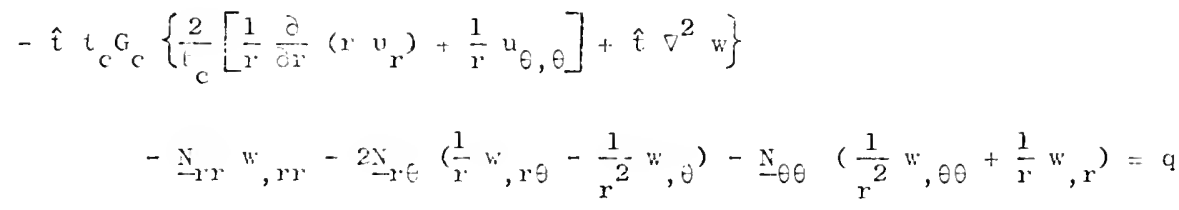
$2 c_{c}\left[\frac{2}{t} u_{v}+\hat{t} w_{, r}\right]-\frac{2 f_{f} f}{1-v_{f}^{2}}\left[\nabla^{2} u_{r}-\frac{1}{r^{2}} u_{r}+\left(\frac{v_{f}-3}{2 r^{2}}\right) u_{\theta, \theta}\right.$

$$
\left.+\frac{\left(1+\nu_{f}\right)}{2 r}\left(u_{\theta, r \theta}-\frac{1}{r} u_{r, \theta \theta}\right)\right]=0
$$

$$
\begin{aligned}
& 2 G_{C}\left[\frac{2}{t} u_{\theta}+\frac{\hat{t}}{r} u_{\theta}\right]-\frac{t_{f}^{E} f}{1 \cdots v_{f}^{2}}\left[\left(1-u_{f}\right)\left(\nabla^{2} u_{\theta}-\frac{1}{r} \cdot u_{\theta}\right)\right. \\
& \left.+\left(1+\nu_{f}\right)\left(\frac{1}{r^{2}} u_{\theta, \theta \theta}+\frac{1}{r} u_{r, r \theta}\right)+\frac{\left(3-r_{f}\right)}{r^{2}} u_{\left.r, \theta_{-}\right]}\right]=0
\end{aligned}
$$

and boundary conditions, 


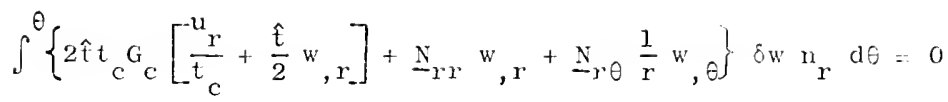

$$
\begin{aligned}
& \int\left\{\frac{\theta}{2 t_{f} E_{f}}\left[u_{r}^{2}\left[i_{r}, r+\nu_{f}\left(\frac{1}{r} u_{\theta, \theta}+\frac{1}{r} u_{r}\right)\right]\right\} u_{r}{ }_{r}{ }_{r} d \theta=0\right. \\
& \int^{\theta}\left\{\frac{f_{f}^{F} r}{1+v_{f}}\left[\frac{1}{r} u_{r, \theta}-\frac{1}{r} u_{\theta}+u_{\theta, r}\right]\right\} \varepsilon u_{\theta} n_{r} d \theta=0 \\
& \text { where } \quad \nabla^{2}=\frac{\partial^{2}}{\partial r^{2}}+\frac{1}{r} \frac{\partial}{\partial r}+\frac{1}{r^{2}} \frac{\partial^{2}}{\partial \theta^{2}}
\end{aligned}
$$

Since only complete ammuar or circular regions are to be considered, the use of continuous eyclical functions of $\theta$ eliminates the need to specify boundary conditions along a radial boundary.

In the present work, which investigates the buckling of annular sandwich panels, the transverso loading function, $q$, is not considered. Such a restriction does not limit the application of the obtained results, since, for snall deflections, the transverse loading function does not inf? vence the buckling load [3].

Finally; as a result of uniform compression along the inner and (or) outel boundaries, an axisymetric buckling mode is assuned (B3) tr result from the lowest critical axial load. Such an assmmption has beer siom by olsson [12] to be valid for single-layer ammular panels.

The governing equations thus becone: 


$$
\begin{aligned}
& -\hat{t} t_{c}{ }_{c}\left\{\frac{2}{t_{c}}\left[\frac{1}{r} \frac{d}{d r}(r u)\right]+\hat{t} \nabla^{2} w\right\}-\frac{1}{r} \frac{d}{d r}\left(r+\frac{N}{r} r \frac{d w}{d r}\right)=0 \\
& 2 G_{C}\left[\frac{2}{t_{c}} u+\hat{t} \frac{d w}{d r}\right]-\frac{2 t_{f} s}{1-v_{f}^{2}}\left[\frac{d^{2} u}{d r}+\frac{1}{r} \frac{d u}{d x}-\frac{u}{r^{2}}\right]=0
\end{aligned}
$$

$$
\text { winere } \nabla^{2}=\frac{1}{r} \frac{d}{d r} \cdot\left(r \cdot \frac{d}{d r}\right)
$$

and,

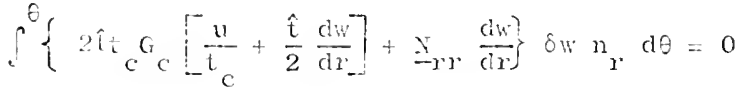

$$
\begin{aligned}
& \int\left\{\frac{d u}{d r}+v_{x} \frac{u}{r}\right\} \varepsilon u n r_{r} d \theta=0
\end{aligned}
$$

In equations (52-55) the subscript $r$ has been omitted from thec displacemont $u$, without conrusion. Also, Nog has been elininated by considering the pre-buclising cquilibrim of the face layers:

$$
\frac{d x r}{d x}+\frac{N_{r}-N g A}{x}=0
$$

\section{Anial Simers Distrintion}

For two-cignensional axisymetric stress distribution in polor cooplunatro, the foverning equat ions are [17]:

$$
\begin{aligned}
& \frac{d^{2} u}{d u^{2}}+\frac{3}{y} \frac{d \vec{u}}{d u}-\frac{\vec{u}}{y^{2}}=0 \\
& x_{-r}=\frac{2 ! d i}{1-v_{f}^{2}}\left(\vec{u}+v_{f} \frac{\vec{u}}{r}\right)
\end{aligned}
$$




$$
N_{\theta \theta}=\frac{2 t_{\hat{i}} E_{f}}{1-\nu_{f}^{2}}\left(\frac{\bar{u}}{r}+\nu \frac{d \bar{u}}{d r}\right)
$$

where $\ddot{u}$ is the pre-buckling lateral displacement.

The general solution of equation (57) is:

$$
\bar{u}=C_{5} r+r_{6}^{\prime r}
$$

where $C_{5}$ and $C_{6}$ are arbitrary constants of integration. Substituting (60) into (5s) yiєldis:

$$
N_{Y Y}=\frac{2 t_{f} E_{f}}{1-\nu_{f}^{2}}\left[C_{5}\left(1+\nu_{f}\right)-C_{6} \frac{\left(1-\nu_{f}\right)-}{r^{2}}\right]
$$

For the case of an anmular panel subjected to uniform conpression along the inmer and outer boundaries (sef Figure 2), boundary conclitions are:

$$
\begin{aligned}
& N_{-r}(a)=-N_{i} \\
& N_{r}(b)=-N_{0}
\end{aligned}
$$

where a enri b are inner and outer radii, respectively, and $\mathrm{N}_{i}$ and $N_{0}$ are ilincr and outcr compressjve forces per unit length, respectively. Imposjng bomdary conclitions (62) on equation (61); we obtain the following axial stress distributjon:

$$
\begin{aligned}
& N_{r r}=\frac{D}{r^{2}}-\mathrm{E} \\
& N_{0 \theta}=-\frac{1}{r^{2}}-F
\end{aligned}
$$




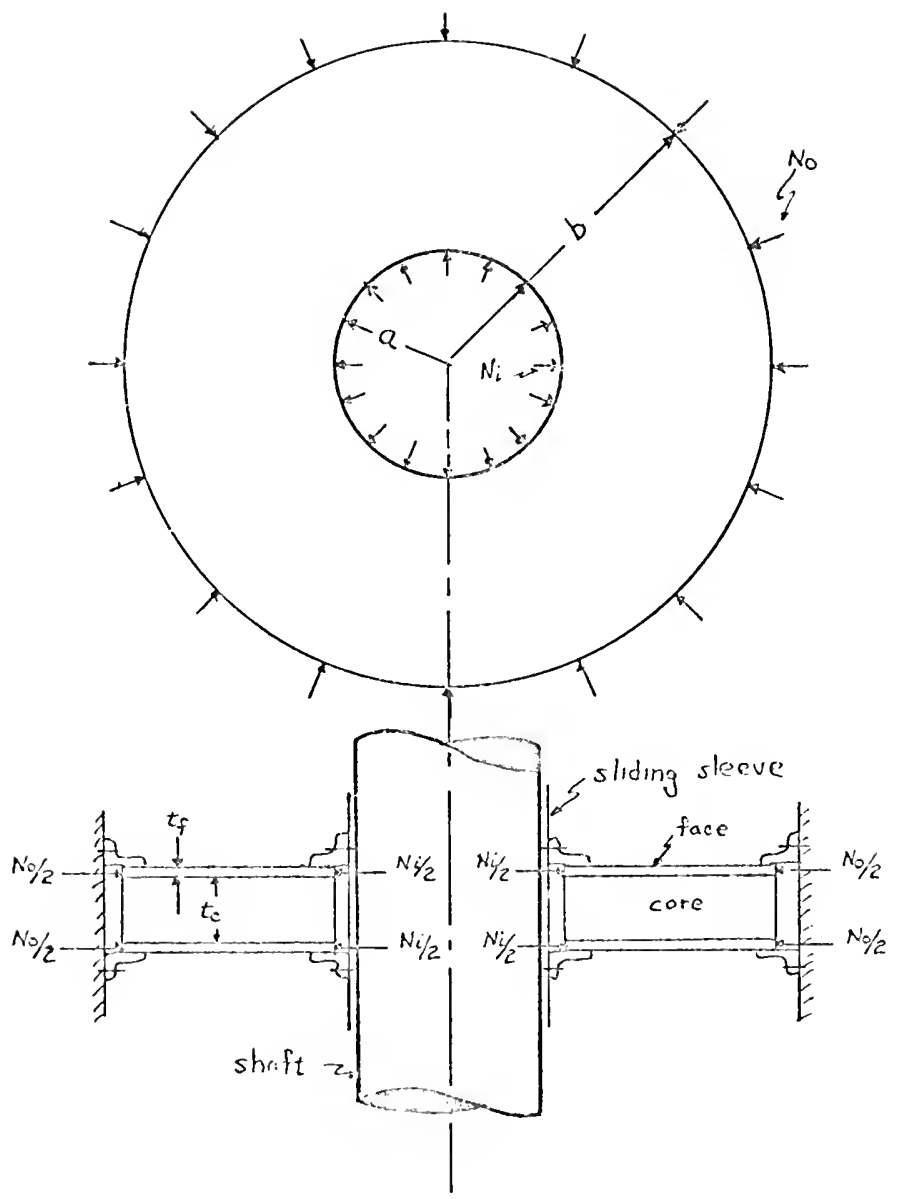

Fignue 2. Annular Sandwich Panel 
where,

$$
D=\frac{b^{2} \beta^{2}\left(N_{O}-N_{i}\right)}{3-\beta^{2}} ; \quad E=\frac{N_{O}-\beta^{2} N_{i}}{1-\beta^{2}}
$$

and,

$$
\beta=\frac{a}{b}
$$

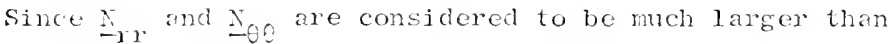
the forces produced by bonding during buckling, the axial stress distribution renajns essentially unchangod during buckling.

$$
\text { Also, if } a=\hat{F}=0 \text {, or } N_{i}=N_{0} \text {, it is casily verified that }
$$

equations (63) and (6.1) reduce to:

$$
N_{r}=N_{\theta}=-x_{0}
$$

\section{Reduced Fquilibrium kquations}

From chapter $1 \mathrm{~V}$, Section 2 , the cquilibrium equations

(reproduced here for convenience) are:

$$
\begin{aligned}
& -\hat{t} t_{C} C_{C}\left\{\frac{2}{t}\left[\frac{1}{r} \frac{d}{d r}(r i)\right]+\hat{t} \nabla^{2} w\right\}-\frac{1}{r} \frac{d}{d r}\left(r N_{r}^{N} \frac{d w}{d r}\right)=0 \\
& 2 G_{c}\left[\frac{2}{t_{c}} u+\hat{t} \frac{d w}{d l_{-}}\right]-\frac{2 t_{f} f}{3-u_{f}^{2}}\left[\frac{d^{2} u}{d r^{2}}+\frac{1}{r} \frac{d u}{d r}-\frac{u}{r^{2}}\right]=0
\end{aligned}
$$

Zajd [9] and Huang and bbeioglu [10] uncomp]e sini? ar equations by operating on the sccond with the linear operator

$$
I_{i}(\varphi)=\frac{1}{x} \frac{d}{d r}(x p)
$$


and elininating the first large common bracket in both equations. The resulting equation is thon directly integrated to yield:

$$
u=\frac{1-v_{f}^{2}}{t_{f} t_{c} \hat{t}_{f}}\left[-\frac{1}{r} \int r \int N_{r r} \cdot \frac{d w}{d r} d r d r+\frac{C_{1}^{\prime}}{2}\left(r \ln r-\frac{r}{2}\right)+\frac{C_{2}^{\prime}}{2} r+\frac{C_{3}^{\prime}}{r}\right]
$$

where $c_{1}^{\prime}, c_{2}^{\prime}$, anc $c_{3}^{\prime}$ are arbitrary constants of integration.

Equation (GS) agrees with Huang and Ebcioglu's results if $N_{r y}$ is defined by equation (67) instead of (63). It should be noted, however, that the uncoupling procedure described above yiej.ds five constants of integration, while only four boundary conditions are available. This is a dircet result of the uncoupling procedure wich initially increases the order of equation (53).

As a consequence of the above-mentioned complications and other considerations which will be discussed later, a different technique is employed in the present worls to uncouple equations (52) and (53).

Multiplying equation (52) by $r$ and integrating directly without the aid of equation (53), we obtain:

$$
u=-\left[\frac{\hat{t} t_{c}}{2}+\frac{N_{2} \cdot}{2 \hat{\imath} G_{c}}\right] \hat{\gamma}+\frac{C_{1}}{2 \hat{t}_{G}} \frac{1}{r}
$$

were,

$$
\varphi=\frac{\mathrm{d} w}{\mathrm{dr}}
$$

and $C_{1}$ is an arbitrary constant of ijiegratjon. 
Comparing equation (69) with equation (68), the advantage of simplicity becomes inmediately evident. Also, il will be made clear in the following sections that the present procedure facilitates the application of boundary conditions, and suggests analogies between the classical single-layer plate thcory and the present analysis of sandwicil panels.

Substituting equation (69) into equation (53), with the aid of (63) and (70), we obtain, after some simplification:

$\left(r^{2}+G\right) r^{2} \frac{d^{2} Q}{d r^{2}}+\left(r^{2}-3 G\right) r \frac{d p}{d r}+\left(H r^{4}-I r^{2}+3 G\right) \varphi=-\frac{B_{1} r^{3}}{F}$

where,

$$
\begin{aligned}
& \mathrm{G}=\frac{\mathrm{D}}{2 \mathrm{~F}} ; \quad \mathrm{H}=\frac{\mathrm{BE}}{\mathrm{F}} ; \quad \mathrm{I}=\frac{\mathrm{F}+\mathrm{BI})}{\mathrm{F}} ; \quad \mathrm{F}=\mathrm{A}-\frac{\mathrm{E}}{2} \\
& A=\frac{t_{C} \hat{t}^{2} G_{c}}{2} ; \quad B=\frac{G_{c}\left(I-v^{2}\right)}{t_{f} t E_{f}}
\end{aligned}
$$

and I and $\mathrm{E}$ are deined by equation (65).

Equations (69) and (71) represent the uncoupl cel equilibrim equations. Since (71) is a second orcier differential equation, two constants of intoration are generated. Togethei with $\mathrm{C}_{1}$ and the constant introduced through the integration of $(70)$, we have four arbitrary constants of interation to be cietermined by boundary conditions at the inser and outer ciges of the amnular panel. 


\section{Bounclary Conditions}

From Chapter Ir, Section 2, the boundary conditions (reprocluced here for convenience) are:

$$
\begin{aligned}
& \int^{\theta}\left\{2 \hat{t} t_{c} G_{c}\left[\frac{u}{t_{c}}+\frac{\hat{t}}{2} \frac{d w}{d r}\right]+N_{r} \cdot \frac{d w}{d r}\right\} \delta w n_{r} d \theta=0 \\
& \int^{\theta}\left\{\frac{d u}{d r}+v_{f} \frac{u}{r}\right\} \delta u_{r} d \theta=0
\end{aligned}
$$

Integral (5.1) requires the specification of either the transverse deflection $w$, or the resultant shear stress along the imer and outer edgcs. Sinilarly, intcoral (55) roguires the sperification of either the relative lateral nowement of one face with respect to the other, or the monent prochecd by tensile stresses on one face and compressive stresses on the other, along the inner and outer edfes. Since we are neglecting the bending rigidity of the faces, the large bracket in integral (55) represents the total edge moment, while u becomes analogous to tire slope, $\varphi$, used in the formulation of boundary conditions for the classical theory of single-layer circular plates [3]. Specifically, in the present work, integrals (51) and (55) are satisfied hrough the following choice of boundary conditions at the inner and outer edges:

$$
\begin{aligned}
& \text { At } u=a: \quad u+\left[\frac{\hat{\mathrm{t} t} \mathrm{c}}{2}+\frac{\hat{N}_{r}}{2 \hat{\mathrm{t}} \mathrm{c}}\right] \mathrm{c}=0 ; \quad \mathrm{u}=0 \\
& \text { At } x=b: \quad w=0 \quad ; \quad u=0
\end{aligned}
$$


Conditions (75) are analogous to bounclary conditions termed clamped or built-in in the classical theory, while conclitions (74), termed "slider" in the present work, have previously been emjoyed for stability analysis of sandwich columns [1] and single-layer annular pane]s [12]. Physically, such a restriction coulc be approximated by allowing a shaft or rigid cylinder to occupy the central hole (see Figure 2).

As the inner radius, a, shrinks to zero, conditions (74) become identical to bounclary conditions present at the center of a circular sandich panel, witbout central hole, constrained along the outer edre only. This limiting proces:; provides a check for our final results since the stability problem associated with such a panel yjelds a relatively simple solution.

\section{Comparison with Other Theories}

Comprison of the present work with existing theories can be facilitated through the use of Table 1. Referring to this table, an analogy between single-layer theory and sandwich theory is observed. Meissner's [11] equation, which can be solved by means of bessel functions, is reduced to a homgeneous differential equation (l: =0) if the shear resultant is nade to vanish at any arbitrary radius. This becomes imnediately evident if we compare the boundary conclition which specifies zero shear with the second form of the equilibrim equation. Consequently, since the shear at the centor of a circular pane] must varist pecause of symmetry, Tinoshouko's [3] bessel cinattion of ordes one is a homogencous differential ecquation. 


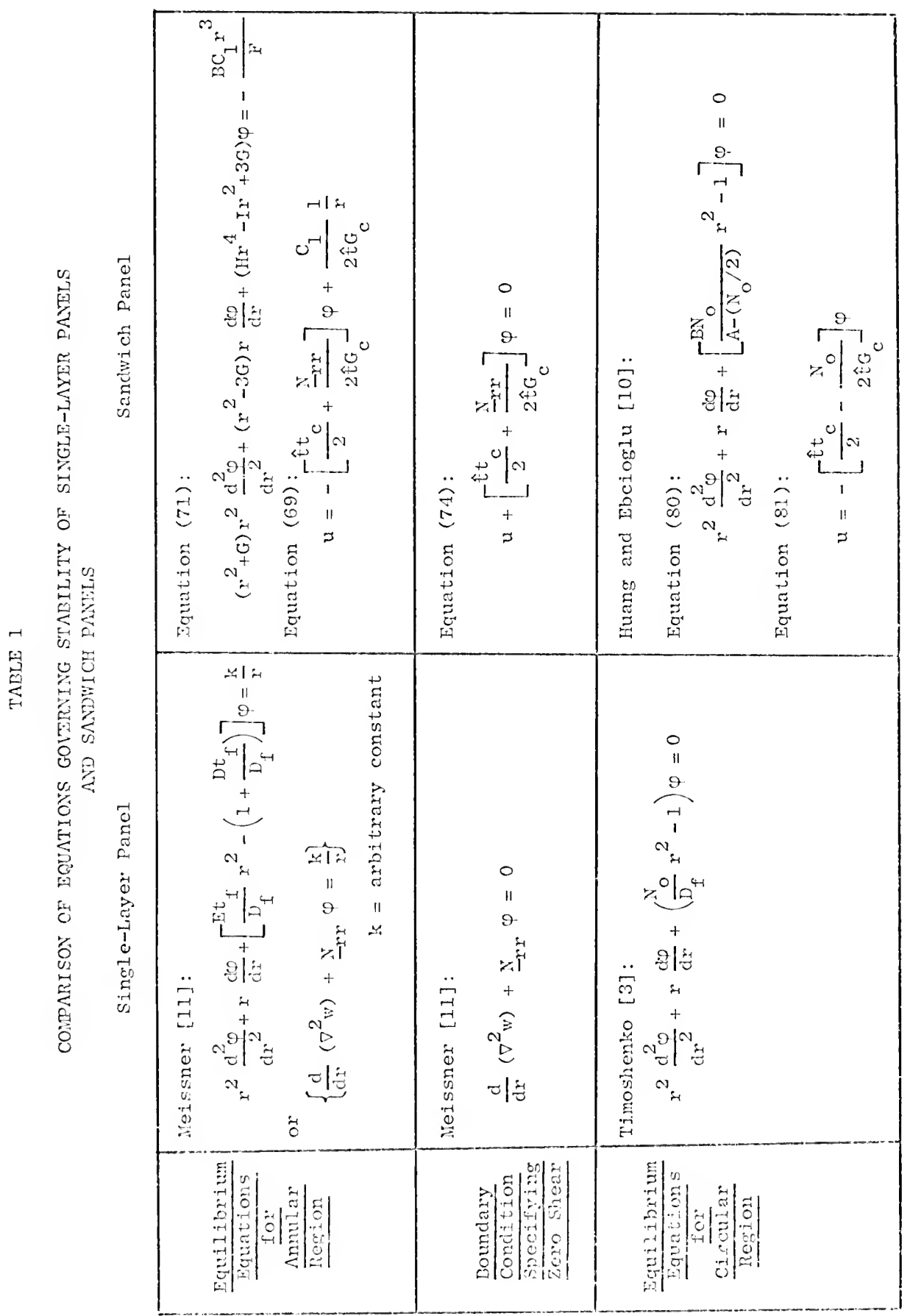


Analogously, one of two equilibrium equations describing an annular region for a sandwich panel reduces to a homogeneous equation $\left(C_{1}=0\right)$ if the shear resultant is specified to vanish at sone arbitrary radius. Such a simplification also provices a direct correspoxience between $u$ and $p$ (see equation (69)). In the case of a circular sandwich paneJ, a homogencous equation again resulis as a consequence of the symetry inrolved, and one of the two equilibrium equations yields bessel functions of orcler one, as is the case in the single layer theory.

The second equilibrium equation for a circular sandwich panel, which is attributed to luang and Ebciog]u, is deduced from equation (69) rather than the original form in which it appeared (see equation (68)). Consequently, without entoloying the present uncoupling technique, the above stated anaiogies rould not be evident.

Since the present work deals with an annular sandwich panel, it becomes obvious that hessel functions cannot be enployed unless $N_{0}=N_{i}$ (see lquations (65) and (67)). Solutions for this special case and the nore general case are obtained in Chaptar v. 
CHAPTER $V$

AXISYMETRIC BUCKLING OF ANNULAR SANDWICH PAYELS

1. Uniform Axial Stress Mistribution

If the pressure along the inner edge of the anmlax sandwich panel is equal to the pressire along the outer edge, or:

$$
N_{i}=N_{0}
$$

then, from (65), (67) and (72):

$$
\begin{aligned}
& \mathrm{D}=\mathrm{G}=\mathrm{O} ; \mathrm{I}=\mathrm{I} ; \quad \mathrm{E}=\mathrm{N}_{\mathrm{O}} \\
& \mathrm{N}_{-\mathrm{P}}=-\mathrm{N}_{\mathrm{O}} ; \quad \mathrm{F}=\mathrm{A}-\frac{\mathrm{N}}{2} ; \mathrm{H}=\frac{\mathrm{BN}}{\mathrm{A}-\left(\mathrm{N}_{0} / 2\right)}
\end{aligned}
$$

which reduces equations (71) and (69) to:

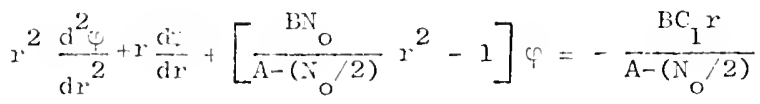

$$
\begin{aligned}
& u=-\left[\frac{\hat{t} \mathrm{c}}{2}-\frac{\mathrm{N}_{\mathrm{O}}}{2 \hat{\mathrm{tG}}}\right] \psi+\frac{\mathrm{C}_{\mathrm{c}}}{2 \hat{\mathrm{tG}}_{\mathrm{c}}} \frac{1}{\mathrm{r}}
\end{aligned}
$$

Applyiug the first of boundary concitions (74) to equation (79) immediately rediuces (78) and (79) to:

$$
\left.r^{2} \frac{d^{2} x}{d r^{2}}+r \frac{d p}{d r}+\left[\frac{b^{x}{ }^{2}}{A-\left(A_{0}-2\right)}\right]\right\}=0
$$




$$
u=-\left[\frac{\hat{t} \mathrm{c}}{2}-\frac{\mathrm{N}_{\mathrm{O}}}{2 \hat{\mathrm{tg}}_{\mathrm{c}}}\right] \varphi
$$

Since (80) is Bessel's equation of order one, a general solution of equations (80) and (81) takes the following form [1S]:

$$
\varphi=A_{1} J_{1}\left[\left(\frac{B N_{0}}{A-\left(N_{0} / 2\right)}\right)^{\frac{1}{2}} r\right]+A_{2} Y_{1}\left[\left(\frac{B N o}{A-\left(N_{0} / 2\right)}\right)^{\frac{1}{2}} r\right]
$$

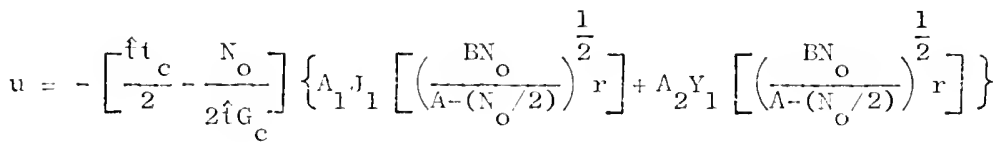

where $J_{1}$ and $\mathrm{Y}_{1}$ are bessel functions of order one of the first and second kind, respectively, and $A_{1}$ and $A_{2}$ are arbitrary constants of interration.

If we now imose the remander of houndary conditions (7i) and $(i 5)$ on equations (82) and ( 83$)$, we have:

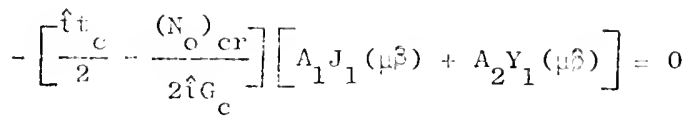

$$
\begin{aligned}
& -\left[\frac{\hat{t} \mathrm{c}}{2}-\frac{\left(\mathrm{N}_{0}\right)_{c I}}{2 \hat{i}_{\mathrm{G}}}\right]\left[\mathrm{A}_{1} \mathrm{~J}_{1}(\mu)+\mathrm{A}_{2} \mathrm{Y}_{1}(\mu)\right]=0
\end{aligned}
$$

where

$$
u=\left(\frac{B\left(\pi_{0}\right) c r}{A-\left[\left(\Omega_{0}\right)\left(r^{\prime}\right]\right.}\right)^{\frac{1}{2}} b ; \beta=\frac{a}{b}
$$


These equations can be satisfied by taking $A_{1}=A_{2}=0$. Then tire deflection at each point of the panel is zero and we obtain the trivial, undeflected form of equilibriun of the panel. The buckling form of equilibrim of the panel becomes possible only if equations (81) yield values for $A_{1}$ and $A_{2}$ different from zero, which recuires that the determinant of the coefficients of these constants vanish. Therefore, after multiplying by $\left(4 / t_{\epsilon} \hat{t}\right)$ and consiciering the first of equations ( 73$)$, our critical condition becomes:

$$
\left[\left(\frac{N}{\mathrm{~A}}\right)_{\mathrm{c} \Gamma}-2\right]^{2}\left[J_{1}(1,) \mathrm{Y}_{1}(\mu \hat{\jmath})-J_{1}(\mu \beta) \mathrm{Y}_{1}(\mu)\right]=0
$$

Equation (86) closely resembles the critical condition obtained by Olsson [12] for a sing] e-layer panel constrained in a similar manner. For a given value of $\beta$, the smallest corresponcling value of $\mu$ for which the second large bracket in equation (86) vanishes is given in table 2 helow [12].

TABLE 2

I,ONEST VALUE OF $\mu$ SATISFYING EQUATION (86)

\begin{tabular}{|c|c|c|c|c|c|}
\hline$\mu$ & $\begin{array}{l}0.0000 \\
3.8 .32\end{array}$ & $\begin{array}{l}0.0256 \\
3.840\end{array}$ & $\begin{array}{l}0.0520 \\
3.860\end{array}$ & $\begin{array}{l}0.0909 \\
3.921\end{array}$ & $\begin{array}{l}0.1000 \\
3.9 .12\end{array}$ \\
\hline$\mu$ & $\begin{array}{l}0.1111 \\
3.965\end{array}$ & $\begin{array}{l}0.1250 \\
4.000\end{array}$ & $\begin{array}{l}0.1433 \\
4.045\end{array}$ & $\begin{array}{l}0.1667 \\
4.116\end{array}$ & $\begin{array}{l}0.2000 \\
4.235\end{array}$ \\
\hline$\beta$ & $\begin{array}{l}0.2500 \\
4.445\end{array}$ & $\begin{array}{l}0.3333 \\
4.905\end{array}$ & $\begin{array}{l}0.3956 \\
5.355\end{array}$ & $\begin{array}{l}0.5000 \\
6.391\end{array}$ & $\begin{array}{l}0.5161 \\
7.016\end{array}$ \\
\hline $\begin{array}{l}\dot{\vec{c}} \\
\mu\end{array}$ & $\begin{array}{l}0.6285 \\
8.523\end{array}$ & $\begin{array}{c}0.6857 \\
10.175\end{array}$ & $\begin{array}{l}0.7634 \\
13.312\end{array}$ & $\begin{array}{l}0.8333 \\
18.873\end{array}$ & $\begin{array}{c}1.000 \\
\infty\end{array}$ \\
\hline
\end{tabular}


And, from the first of (85):

$$
\left(\frac{N}{A}\right)_{c r}=\frac{\mu^{2}}{b^{2} B+\mu^{2} / 2}
$$

It can be shown that $\left(N_{\mathrm{o}} / \mathrm{A}\right)_{\mathrm{cr}}$ given by equation (87) is a monotonic increasing funciion of $\mu$. Therefore, the lowest value of $\mu$ results in the lowest value of $\left(N_{0} / A\right) C^{*}$ Also, since $b^{2} \mathrm{~B}$ is always positive, equation ( 87$)$ yields values of $\left(\mathrm{N}_{\mathrm{O}} / \mathrm{A}\right) \mathrm{cr}$. which are greater than or equal to two; and we can therefore conclude that the lowest root of equation ( 86 ) is always given by equation (87) in conjunction with Table 2. (See Figure 3.)

The first of boundary conditions (75) has not been used to obtain the above results since the buckling load is independent of a transverse translation of the panel as a whole. In this respect our analysis parallels the classical single-layer theory [3].

For $a=\hat{3}=0$, equation $(87)$ becomes:

$$
\left(\frac{N}{A}\right)_{c r}=\frac{(3.832)^{2}}{b^{2} B+(3.832)^{2} / 2}
$$

Equation (s8) agrees with Huang and Ebcioglu's [10] results if the present rotalion is used. The valjdity of this limiting process was discussed in Scetion 5 of Chapter IV.

As $G_{c}$ appioaches a very large value, the first term in the denominator of equation (87) becomes clominant, and we obiain:

$$
\left(N_{0}\right)_{c r}=\frac{\mu^{2} n^{\prime}}{b^{2}}
$$


where the "effective bending rigidity," $\mathrm{D}^{\prime}$, is defined:

$$
D^{\prime}=\frac{\hat{t}^{2} t^{2} t_{f} E_{f}}{2\left(1-\nu_{f}^{2}\right)}
$$

Equation (89) in conjunction with lable 2 agrees with the results oblajned by olsson [12] for a single-layer annular parel subjected to uniform inmer and outer axial pressures of equal intensity. For $\mathrm{a}=\beta=0$, equation (89) becomes:

$$
\left(N_{0}\right)_{c r}=\frac{(3.832)^{2} D^{\prime}}{b^{2}}
$$

Equation (91) is identical to the buckling load obtained by Timoshento [3] for a circular single-layer ponel clamped along the outer edgre.

This limiting process is intujtively expected, since, for $G_{C}=\infty$, the only structural function of the core is to control the distance between the face layers. A similar relationship cxists between the web and flanges of an I-beam.

\section{Gencral Solution}

We now return to a general solution of equations (69) and (71). Introducing the dimensionless variable, i, through the transformation:

$$
\eta=r / b
$$


equation (71) becomes:

$$
\eta^{2}\left(M \eta^{2}+1\right) \frac{d^{2} \varphi}{d \eta^{2}}+\eta\left(N \eta^{2}-3\right) \frac{d \varphi}{d \eta}+\left(R \eta^{4}-Q^{2} \eta^{2}+3\right) \varphi=-\frac{\mathrm{b}^{2} B C 1 \eta}{G F}
$$

where,

$$
N=\frac{h^{2}}{G} ; \quad R=b^{2} H A ; \quad Q=I N
$$

Sinil $y$, equation (69) becomes:

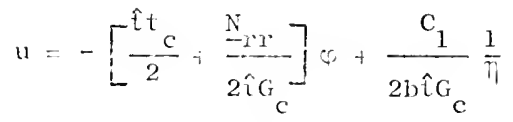

Since we seel: a scries solution of equation (93), and choose to exprnd ouy sories about the point $\uparrow=1$, the following additional transfornation is introduced:

$$
\xi=\eta-1
$$

The reasoris for scoling a solution about the point $\pi=1 \quad(\xi=0)$ will be discussed in sections 5 and 6 of this chapter.

With the aid of equation (96), and after some simplification, equation (93) becones:

$$
\begin{aligned}
{[(N+1)} & \left.+2(2 N+1) \xi+(6 N+1) \xi^{2}+4 N \xi^{3}+N \xi^{4}\right] \frac{\mathrm{d}^{2} \varphi}{\mathrm{d} \xi^{2}}+[(N-3) \\
& \left.+3(N-1) \xi+3 N \xi^{2}+N \xi^{3}\right] \frac{\mathrm{d} \varphi}{\mathrm{d} \xi}+[(\mathrm{R} \cdot \mathrm{Q}+3)+(1 \mathrm{R}-2 \mathrm{Q}) \xi \\
& \left.+(6 \mathrm{R}-Q) \xi^{2}+4 \mathrm{R}^{3}+\mathrm{R}^{4}\right] \varphi=-\frac{\mathrm{b}^{2} \mathrm{BC}(1+\xi)}{\mathrm{GF}}
\end{aligned}
$$


To obtain a complementary solution of equation (97) the following infinite series is employed [18]:

$$
\varphi_{c}=\sum_{k=0}^{\infty} A_{k} \xi^{k}
$$

where the coefficients $A_{k}$ are functions of the clastic and geometric properties of the panel and the critical buckling load. The radius of convergence of series ( 98 ) can be shown (see Section 6, Chapter v) to be of sufficient magnituce for our particular problem.

Substituting (98) into a homogeneous form of equation (97), and collecting coefficients of common powers of $\xi$, we have:

$$
\begin{aligned}
& \left\{\left[2(N+1) A_{2}+(N-3) A_{1}+(R-Q+3) A_{0}\right]+\left[G(N+1) A_{3}+2(5 N-1) A_{2}\right.\right. \\
& \left.+(R-Q+3 N) A_{1}+(1 R-2 Q) A_{0}\right] \xi+\left[12(N+1) A_{4}+(27 X+3) A_{3}\right. \\
& \left.+(R-Q+] B-1) A_{2}+(4 R-2 Q+3 N) A_{1}+(6 R-Q) A_{0}\right] \xi^{2} \\
& +\left[2 \left(N(N+1) A_{5}+4(13 N+3) A_{4}+(45 N+R-Q) A_{3}\right.\right. \\
& \left.+(14 N+1 R-2 Q) A_{2}+(N+6 R-Q) A_{1}+4 R A_{0}\right] 5^{3} \\
& +\cdot \cdot \cdot \cdot \cdot \cdot \cdot \cdot \cdot\}=0
\end{aligned}
$$

lin order that this series vanish for all values of $\xi$ in sone region surromding $\xi=0$, it is necessary and sufficient that the coerficients of each poter of $\xi$ vanish [18]. This produces the folloning relations in which some coeficients have been eliminated though the accumulative introcuction of previously computed coefficients: 


$$
\begin{aligned}
& A_{2}=-\frac{(R-Q+3)}{2(N+1)} A_{0}-\frac{(N-3)}{2(N+1)} A_{1} \\
& A_{3}=\left[\frac{(5 N-1)(R-Q+3)}{6(N+1)^{2}}-\frac{(4 R-2 Q)}{6(N+1)}\right] A_{0}+\left[\frac{(5 N-1)(N-3)}{6(N+1)^{2}}-\frac{(F-Q+3 N)}{6(N+1)}\right] A_{1} \\
& A_{4}=\left[-\frac{(6 R-Q)}{12(N+1)}+\frac{(R-Q+3)(R-Q+18 N-1)}{24(N+1)^{2}}+\frac{(27 N+3)(4 R-2 Q)}{72(N+1)^{2}}\right. \\
& \left.-\frac{(27 N-3)(5 N-1)(\mathrm{R}-\mathrm{Q}+3)}{72(N+1)^{3}}\right] \mathrm{A}_{0}+\left[-\frac{(4 \mathrm{R}-2 Q+3 N)}{12(N+1)}+\frac{(\mathrm{N}-3)(\mathrm{R}-\mathrm{Q}+18 \mathrm{~N}-1)}{24(\mathrm{~N}+1)^{2}}\right. \\
& \left.+\frac{(27 N+3)(R-Q+3 N)}{72(N+1)^{2}}-\frac{(27 N+3)(5 N-1)(N-3)}{72(N+1)^{3}}\right] A_{1} \\
& A_{5}=\left[\frac{(13 N+3)(6 \mathrm{R}-\mathrm{Q})}{60(\mathrm{~N}+1)^{2}}-\frac{(13 \mathrm{~N}+3)(\mathrm{R}-\mathrm{Q}+3)(\mathrm{R}-\mathrm{Q}+18 \mathrm{~N}-1)}{120(\mathrm{~N}+1)^{3}}\right. \\
& -\frac{(13 N+3)(27 N+3)(1 \mathrm{k}-2 \mathrm{Q})}{360(\mathrm{~N}+1)^{3}}+\frac{(13 \mathrm{~N}+3)(27 \mathrm{~N}+3)(5 \mathrm{~N}-1)(\mathrm{R}-\mathrm{Q}+3)}{360(\mathrm{~N}+1)^{4}} \\
& -\frac{(45 N+R-Q)(5 N-1)(R-Q+3)}{120(N+1)^{3}}+\frac{(45 N+R-Q)(4 \mathrm{R}-2 \mathrm{Q})}{120(N+1)^{2}}+\frac{(R-Q+3)(14 N+4 R-2 Q)}{40(N+1)^{2}} \\
& \left.-\frac{4 R}{20(N+1)}\right] A_{0}+\left[\frac{(13 N+3)(4 R-2 Q+3 N)}{60(N+1)^{2}}-\frac{(13 N+3)(N-3)(R-Q+18 N-1)}{120(N+1)^{3}}\right. \\
& -\frac{(13 N+3)(27 N+3)(N-Q+3 N)}{360(N+1)^{3}}+\frac{(13 N+3)(27 N+3)(5 N-1)(N-3)}{360(N+1)^{4}} \\
& -\frac{(45 N+R-Q)(5 N-1)(N-3)}{120(N+1)^{3}}+\frac{(45 N+R-Q)(R-Q+3 N)}{120(N+1)^{2}} \\
& \left.+\frac{(N-3)(i \cdot 1 N+4 R-2 Q)}{40(N+1)^{2}}-\frac{(N+6 R-Q)}{20(N+1)}\right] A_{1}
\end{aligned}
$$

$$
A_{k}=
$$

vhere $A_{0}$ ani $A_{1}$ remain arbitrary. 
Because of the complexity of the computations, and the imnediate requirements, no recursion formula is sought in the present analysis.

With the aid of (100-103), our complementary solution becomes:

$$
\begin{aligned}
& \varphi_{C}(\xi)=A_{O}\left\{1-\frac{(R-Q+3)}{2(N+1)} \xi^{2}+\left[\frac{(5 N-1)(R-Q+3)}{6(N+1)^{2}}-\frac{(4 R-2 Q)}{6(N+1)}\right] \xi^{3}\right. \\
& +\left[-\frac{(6 R-Q)}{12(N+1)}+\frac{(R-Q+3)(R-Q+18 N-1)}{2+(N+1)^{2}}+\frac{(27 N+3)(4 R-2 Q)}{72(N+1)^{2}}\right. \\
& \left.-\frac{(27 N+3)(5 N-1)(R-Q+3)}{72(N+1)^{3}}\right] \xi^{4}+\left[\frac{(13 N+3)(6 R-Q)}{60(N+1)^{2}}\right. \\
& -\frac{(13 X+3)(R-Q+3)(N-Q+18 N-1)}{120(N+1)^{3}}-\frac{(13 N+3)(27 N+3)(4 R-2 Q)}{360(N+1)^{3}} \\
& +\frac{(13 N+3)(27 N+3)(5 N-1)(R-Q+3)}{360(N+1)^{4}}-\frac{(15 N+R-Q)(5 N-1)(R-Q+3)}{120(N+1)^{3}} \\
& \left.\left.+\frac{(45 N+R-Q)(4 R-2 Q)}{120(N+1)^{2}}+\frac{(R-Q+3)(14 N+4 R-2 Q)}{40(N+1)^{2}}-\frac{4 R}{20(N+1)}\right] \xi^{5}+\cdot \cdot \cdot\right\} \\
& +A_{1}\left\{\xi-\frac{(N-3)}{2(N+1)} \xi^{2}+\left[\frac{(5 N-1)(N-3)}{6(N+1)^{2}}-\frac{(R-Q+3 N)}{6(N+1)}\right] \xi^{3}\right. \\
& +\left[-\frac{(1 R-2 Q+3 N)}{12(N+1)}+\frac{(N-3)(R-Q+18 N-1)}{24(N+1)^{2}} \div \frac{(27 N+3)(N-Q+3 N)}{72(N+1)^{2}}\right. \\
& \left.-\frac{(27 N+3)(5 N-1)(N-3)}{72(N+1)^{3}}\right] \xi^{4}+\left[\frac{(13 N+3)(4 R-2 Q+3 N)}{60(N+1)^{2}}\right. \\
& -\frac{(13 N+3)(N-3)(R-Q+18 N-1)}{120(N+1)^{3}}-\frac{(13 N+3)(27 N+3)(N-Q+3 N)}{360(N+1)^{3}} \\
& +\frac{(13 N+3)(27 N+3)(5 N-1)(N-3)}{360(N+1)^{4}}-\frac{(45 N+R-Q)(5 N-1)(N-3)}{120(N+1)^{3}} \\
& \left.+\frac{(45 N+R-Q)(R-Q+3 N)}{120(N+1)^{2}}+\frac{(N-3)(1+N+4 R-2 Q)}{40(N+1)^{2}}-\frac{(N+6 R-Q)}{20(N+1)}\right] \xi^{5} \\
& +\cdot \cdot \cdot \cdot \cdot \cdot \cdot\}
\end{aligned}
$$


Following a procedure similar to tinat enployed in the previous section, equations (95) and (104), together with a particular solution of equation (97), are constrained according to boundary conditions (74) and (75).

Imposing the first of boundary conditions (74) on equation (95) eliminates the need to find a particular solution of equation (97), since "e have $C_{1}=0$. The subscript c can therefore be eliminated from equition (98) and our solution becones a complete solution.

Froin equations (92) and (96), we note that at $x=b, \eta=1$ and $5=0$. Therefore, from equation (95), recalling that $C_{1}=0$ and $x_{r r}(b)=-x_{0}$ (see equation $(62)$ ), the second of bounclary conclitionc (75) becomes:

$$
\left[\left(\frac{N}{A}\right)-2\right] \varphi(\xi=0)=0
$$

And since $\left(X_{0} / A\right) \neq 2$ in general, condition (105) imposed on equation (10-1) yields $A_{0}=0$

Sinilarly, the second of houndary conclitions (74), evaluated at $r=$ a $(E=5-1)$, and expressect through equation (95) becomes:

$$
\left[\left(\frac{N_{i}}{A}\right)-2\right] \varphi(\xi=\xi-1)=0
$$

After substituting equation (104) into (106) with $\mathrm{A}_{\mathrm{O}}=0$, and noting that $i n$ general $\left(N_{i} / A\right) \neq 2$, we arrive at the general buching critcria for an amular sanchich panel: 


$$
\begin{aligned}
& A_{1}\left\{(\xi-1)-\frac{(N-3)}{2(N+1)}(\beta-1)^{2}+\left[\frac{(5 N-1)(N-3)}{6(N+1)^{2}}-\frac{(R-Q+3 N)}{6(N+1)}\right](\beta-1)^{3}\right. \\
& +\left[-\frac{(4 R-2 Q+3 N)}{12(N+1)}+\frac{(N-3)(R-Q+18 N-1)}{24(N+1)^{2}}+\frac{(27 N+3)(R-Q+3 N)}{72(N+1)^{2}}\right. \\
& \left.-\frac{(27 N+3)(5 N-1)(N-3)}{72(N+1)^{3}}\right](\beta-1)^{4}+\left[\frac{(13 N+3)(4 R-2 Q+3 N)}{60(N+1)^{2}}\right. \\
& -\frac{(13 N+3)(N-3)(R-Q+18 N-1)}{120(N+1)^{3}}-\frac{(13 N+3)(27 N+3)(R-Q+3 N)}{360(N+1)^{3}} \\
& +\frac{(13 X+3)(27 X+3)(5 X-1)(X-3)}{360(X+1)^{4}}-\frac{(45 X+1 i-Q)(5 N-1)(X-3)}{120(N+1)^{3}} \\
& \left.+\frac{(45 N+R-Q)(R-Q+3 N)}{120(N+1)^{2}}+\frac{(N-3)(14 N+1 R-2 Q)}{40(N+1)^{2}}-\frac{(N+6 R-Q)}{20(N+1)}\right](-1)^{5} \\
& +\cdot \cdot \cdot \cdot \cdot \cdot \cdot\}=0
\end{aligned}
$$

As explained in the previous section, the undeflected form of a panel in equilibrium, j.e., when $A_{1}=0$, is of jittle interest. Therefore, approximate clitical buckljng loads of the panel under discussion may be computed by consiciering a finite number of terms in equation (107).

The first of boundary conditions (75), as stated carlier, is not used in obtaining the above results, since the buckling load is independent of a transverse translation of the panel as a whole. 


\section{Successive Approximations}

From equations (94), (72) and (65), we have:

$$
\begin{aligned}
& N=\frac{2 A\left(1-\beta^{2}\right)}{\beta^{2}\left(N_{0}-N_{i}\right)}-\frac{\left(N_{0}-\beta^{2} N_{i}\right)}{\beta^{2}\left(N_{0}-N_{i}\right)} \\
& R=\frac{2 b^{2} B}{\beta^{2}} \frac{\left(N_{0}-\beta^{2} N_{i}\right)}{\left(N_{0}-N_{i}\right)} \\
& Q=\frac{2 A\left(1-\beta^{2}\right)}{\beta^{2}\left(N_{0}-N_{i}\right)}-\frac{\left(N_{0}-\beta^{2} N_{i}\right)}{\beta^{2}\left(N_{0}-N_{i}\right)}+2 b^{2} B
\end{aligned}
$$

We designate the first approxination to be that which considers only the first term of the infinite series (107), and consecutively add a term for each succeeding approxination. Therefore, with the aid of (108-110), and after some simplification, we have:

\section{Fixst Amproximation}

The first approxination yields no results since the loading functions are not present in the first term of series (107).

Second Approxination

$$
\begin{aligned}
& \frac{4 A\left(1-\hat{\hat{N}}^{2}\right)}{\beta^{2}\left[\left(\mathrm{~N}_{\mathrm{O}}\right)_{\mathrm{Cr}}-\left(\mathrm{N}_{\mathrm{i}}\right)_{\mathrm{Cr}}\right]}-\frac{2\left[\left(\mathrm{~N}_{\mathrm{O}}\right)_{\mathrm{Cr}}-\hat{\beta}^{2}\left(\mathrm{~N}_{\mathrm{i}}\right)_{\mathrm{Cr}}\right]}{\beta^{2}\left[\left(\mathrm{~N}_{\mathrm{O}}\right)_{\mathrm{Cr}}-\left(\mathrm{N}_{\mathrm{i}}\right)_{\mathrm{Cr}}\right]}+2 \\
& -(\beta-1)\left[\frac{2 A\left(1-\beta^{2}\right)}{\beta^{2}\left[\left(N_{0}\right)_{C r}-\left(N_{i}\right)_{C r}\right]}-\frac{\left[\left(N_{O}\right)_{C r}-\beta^{2}\left(N_{i}\right)_{C r}\right]}{\beta^{2}\left[\left(N_{O}\right)_{C r}-\left(N_{i}\right)_{C r}\right]}-3\right]=0
\end{aligned}
$$




$$
\begin{aligned}
& 6\left[\frac{2 A\left(1-S^{2}\right)}{\beta^{2}\left[\left(N_{0}\right)_{c r}-\left(N_{i}\right)_{c r}\right]}-\frac{\left[\left(N_{0}\right) c^{\left.-\beta^{2}\left(N_{i}\right)_{c r}\right]}\right.}{\beta^{2}\left[\left(N_{o}\right)_{c r}-\left(N_{i}\right)_{c r}\right]}\right]^{2}-3\left[\frac{2 A\left(1-\beta^{2}\right)}{\beta^{2}\left[\left(N_{0}\right)_{c r}-\left(N_{i}\right) c r\right]}\right. \\
& \left.-\frac{\left[\left(N_{0}\right)_{c r} \beta^{2}\left(N_{i}\right)_{c r}\right]}{\beta^{2}\left[\left(N_{0}\right)_{c 1}-\left(N_{i}\right)_{c r}\right]}+1\right]\left[\frac{2 A\left(1-\beta^{2}\right)}{\beta^{2}\left[\left(N_{0}\right)_{c r}-\left(N_{i}\right)_{c r}\right]}-\frac{\left[\left(N_{0}\right)_{c r}-\beta^{2}\left(N_{i}\right)_{c r}\right]}{\beta^{2}\left[\left(N_{0}\right)_{c r}-\left(N_{i}\right)_{c r}\right]}-3\right](\beta-1) \\
& +\left[\frac{10 A\left(1-\hat{\beta}^{2}\right)}{\beta^{2}\left[\left(N_{0}\right)_{C r}-\left(N_{i}\right)_{c r}\right]}-\frac{5\left[\left(N_{0}\right)_{c r} \beta^{2}\left(N_{i}\right)_{c r}\right]}{\beta^{2}\left[\left(N_{0}\right)_{c r}-\left(N_{i}\right)_{C r}\right]}-1\right]\left[\frac{2 A\left(1-\beta^{2}\right)}{\beta^{2}\left[\left(N_{0}\right)_{c r}-\left(N_{i}\right)_{C r}\right]}-\right.
\end{aligned}
$$

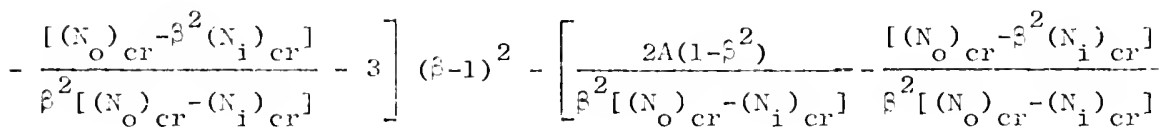

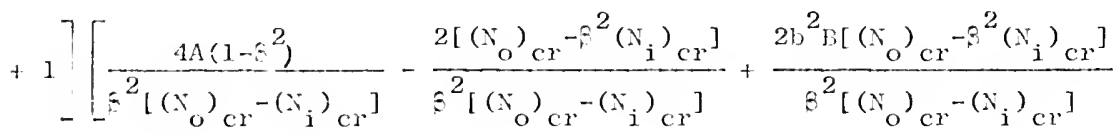

$$
\begin{aligned}
& \left.-2 b^{2} B\right](s-1)^{2}=0
\end{aligned}
$$

Fourth Approximation

$$
\begin{aligned}
& 72\left[\frac{2 A\left(1-\varepsilon^{2}\right)}{\beta^{2}\left[\left(N_{0}\right)_{c r}-\left(N_{i}\right)_{c r}\right]}-\frac{\left[\left(N_{0}\right) c r^{2}\left(N_{i}\right)_{c r}\right]}{8^{2}\left[\left(N_{0}\right)_{c r}-\left(N_{i}\right)_{c r}\right]}+1\right]^{3}
\end{aligned}
$$

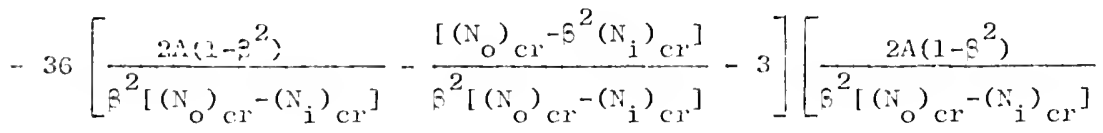

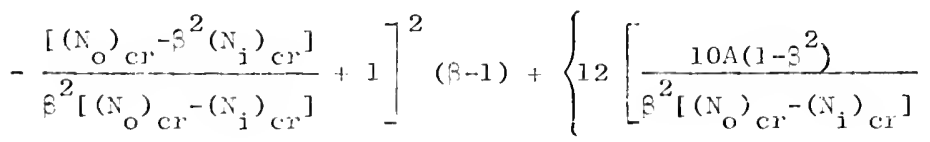


55

$$
\begin{aligned}
& \left.-\frac{5\left[\left(N_{0}\right) c^{-} \beta^{2}\left(N_{i}\right)_{c r}\right]}{\beta^{2}\left[\left(N_{0}\right)_{c r}-\left(N_{i}\right)_{c r}\right]}-1\right]\left[\frac{2 A\left(1-\beta^{2}\right)}{\beta^{2}\left[\left(N_{0}\right)_{c r}-\left(N_{i}\right)_{c r}\right]}-\frac{\left[\left(N_{0}\right)_{c r}-\beta^{2}\left(N_{i}\right)_{c r}\right]}{\beta^{2}\left[\left(N_{0}\right)_{c r}-\left(N_{i}\right)_{c r}\right]}-3\right] \\
& {\left[\frac{2 A\left(1-\beta^{2}\right)}{\beta^{2}\left[\left(N_{0}\right)_{c r}-\left(N_{i}\right)_{c r}\right]}-\frac{\left[\left(N_{0}\right)_{c r}-\beta^{2}\left(N_{i}\right)_{c r}\right]}{\beta^{2}\left[\left(N_{0}\right)_{c r}-\left(N_{i}\right)_{c r}\right]}+1\right]-12\left[\frac{2 A\left(i-\beta^{2}\right)}{\beta^{2}\left[\left(N_{0}\right)_{c r}-\left(N_{i}\right)_{c r}\right]}\right.}
\end{aligned}
$$

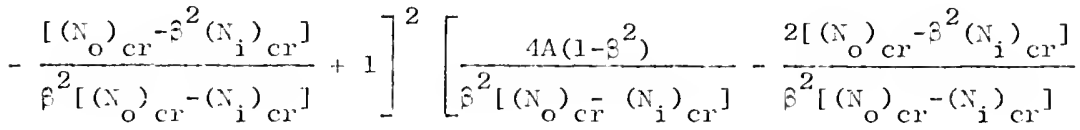

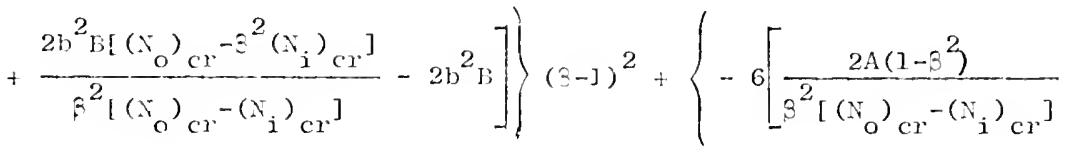

$$
\begin{aligned}
& \left.-\frac{\left[\left(N_{0}\right)_{c r}-\beta^{2}\left(N_{i}\right)_{c r}\right]}{\beta^{2}\left[\left(N_{0}\right)_{c r}-\left(N_{i}\right)_{c r}\right]}+1\right]^{2}\left[\frac{2 A\left(1-\beta^{2}\right)}{\beta^{2}\left[\left(N_{0}\right)_{c r}-\left(N_{i}\right)_{c r}\right]}-\frac{\left[\left(N_{0}\right)_{c r}-\beta^{2}\left(N_{i}\right)_{c r}\right]}{\beta^{2}\left[\left(N_{0}\right)_{C r}-\left(N_{i}\right)_{c r}\right]}\right.
\end{aligned}
$$

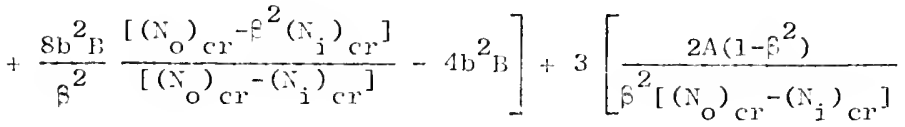

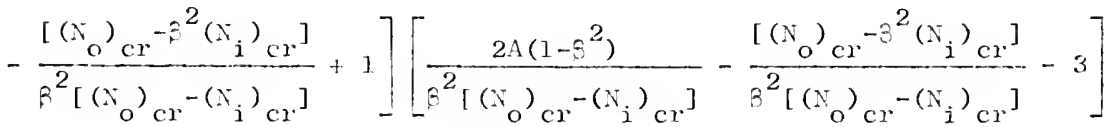

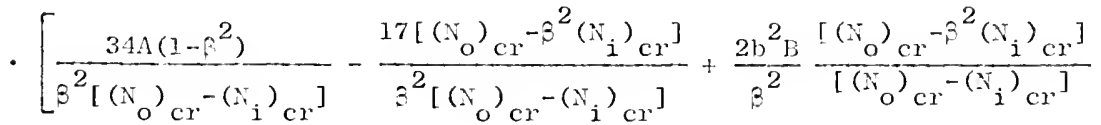

$$
\begin{aligned}
& \left.-2 \mathrm{~b}^{2} \mathrm{~B}-1\right]+\left[\frac{2 A\left(1-\beta^{2}\right)}{\beta^{2}\left[\left(N_{O}\right)_{\mathrm{cr}}-\left(N_{i}\right)_{\mathrm{cr}}\right]}-\frac{\left[\left(N_{\mathrm{O}}\right)_{\mathrm{cr}}-\beta^{2}\left(N_{i}\right)_{\mathrm{cr}}\right]}{\beta^{2}\left[\left(N_{\mathrm{O}}\right)_{\mathrm{cr}}-\left(N_{i}\right)_{\mathrm{cr}}\right]}+1\right]
\end{aligned}
$$

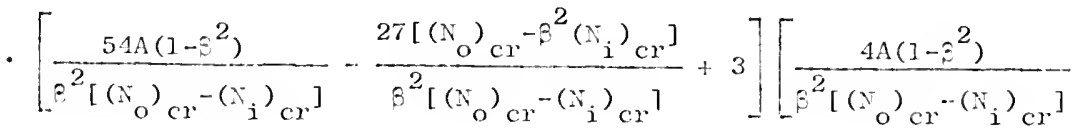


56

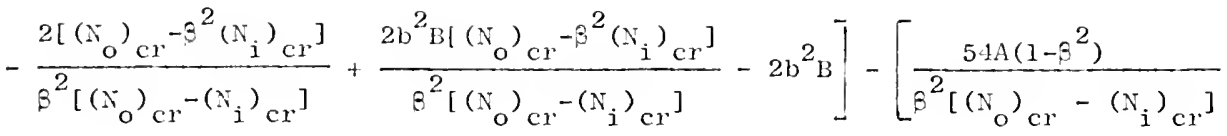

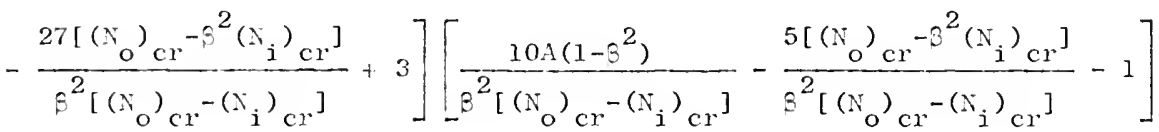

$$
\begin{aligned}
& \left.\cdot\left[\frac{2 A\left(1-\beta^{2}\right)}{\beta^{2}\left[\left(N_{O}\right)_{C r}-\left(N_{i}\right)_{C r}\right]}-\frac{\left[\left(N_{0}\right)_{C r}-\beta^{2}\left(N_{i}\right)_{C r}\right]}{\beta^{2}\left[\left(N_{O}\right)_{C r}-\left(N_{i}\right)_{C r}\right]}-3\right]\right\}(\beta-1)^{3}=0
\end{aligned}
$$

Fifth Approximation

$$
\begin{aligned}
& 360\left[\frac{2 A\left(1-\beta^{2}\right)}{\beta^{2}\left[\left(N_{\mathrm{o}}\right)_{\mathrm{Cr}}-\left(N_{\mathrm{i}}\right)_{\mathrm{Cr}}\right]}-\frac{\left[\left(N_{\mathrm{o}}\right)_{\mathrm{cr}}-\beta^{2}\left(N_{\mathrm{i}}\right)_{\mathrm{cr}}\right]}{\beta^{2}\left[\left(N_{\mathrm{o}}\right)_{\mathrm{Cr}}-\left(\mathrm{N}_{\mathrm{i}}\right)_{\mathrm{Cr}}\right]}+1\right]^{4} \\
& \left.-180 \frac{2 A\left(1-\beta^{2}\right)}{3^{2}\left[\left(N_{0}\right)_{C r}-\left(N_{i}\right)_{C r}\right]}-\frac{\left[\left(N_{0}\right)_{C r}-\beta^{2}\left(X_{i}\right)_{C r}\right]}{3^{2}\left[\left(N_{0}\right)_{C r}-\left(N_{i}\right)_{C r}\right]}+1\right]^{3}\left[\frac{2 A\left(1-\beta^{2}\right)}{\beta^{2}\left[\left(N_{0}\right)_{C r}-\left(N_{i}\right)_{C r}\right]}\right.
\end{aligned}
$$

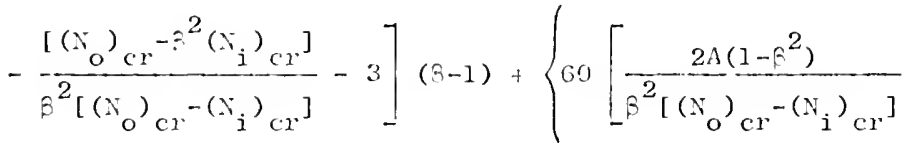

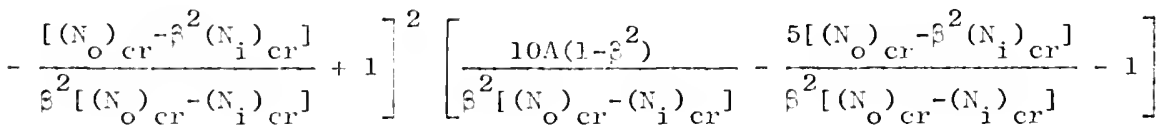

$$
\begin{aligned}
& \cdot\left[\frac{2 A\left(1-\beta^{2}\right)}{\beta^{2}\left[\left(N_{0}\right)_{C r}-\left(N_{i}\right)_{C r}\right]}-\frac{\left[\left(N_{0}\right) c r-\beta^{2}\left(N_{i}\right)_{C r}\right]}{\beta^{2}\left[\left(N_{0}\right)_{C r}-\left(N_{i}\right)_{C r}\right]}-3\right]-60\left[\frac{2 A\left(1-\beta^{2}\right)}{\beta^{2}\left[\left(N_{O}\right)_{C r}-\left(N_{i}\right)_{C r}\right]}\right.
\end{aligned}
$$

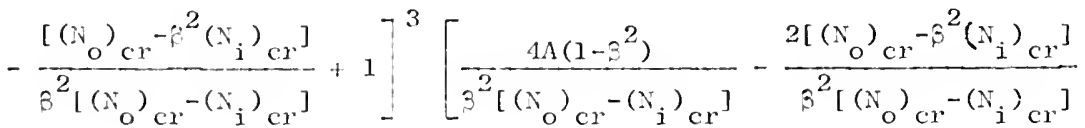


57

$$
\begin{aligned}
& \left.\left.+\frac{2 b^{2} B\left[\left(N_{0}\right) c^{\left.-\beta^{2}\left(N_{i}\right)_{c r}\right]}\right.}{\beta^{2}\left[\left(N_{0}\right)_{c r}-\left(N_{i}\right)_{C r}\right]}-2 b^{2} B\right]\right\}(\beta-1)^{2}+\left\{-30\left[\frac{2 A\left(1-\beta^{2}\right)}{\beta^{2}\left[\left(N_{0}\right)_{C r}-\left(N_{i}\right)_{C r}\right]}\right.\right. \\
& \left.-\frac{\left[\left(N_{0}\right)_{c r}-\beta^{2}\left(N_{i}\right)_{c r}\right]}{\beta^{2}\left[\left(N_{0}\right)_{c r}-\left(N_{i}\right)_{c r}\right]}+1\right]^{3}\left[\frac{2 A\left(1-\beta^{2}\right)}{\beta^{2}\left[\left(N_{0}\right)_{c r}-\left(N_{i}\right)_{C r}\right]}-\frac{\left[\left(N_{0}\right)_{c r}-\beta^{2}\left(N_{i}\right)_{c r}\right]}{\beta^{2}\left[\left(N_{0}\right)_{c r}-\left(N_{i}\right)_{c r}\right]}\right. \\
& \left.+\frac{8 b^{2} E\left[\left(N_{0}\right)_{c r^{-}} \beta^{2}\left(N_{i}\right)_{c r}\right]}{\beta^{2}\left[\left(N_{0}\right)_{c r}-\left(N_{i}\right)_{c r}\right]}-4 b^{2} \mathrm{D}\right]+15\left[\frac{2 A\left(1-\beta^{2}\right)}{\beta^{2}\left[\left(N_{0}\right)_{c r}-\left(N_{i}\right)_{c r}\right]}\right. \\
& \left.-\frac{\left[\left(N_{0}\right) c r^{-} Q^{2}\left(N_{i}\right)_{c r}\right]}{3^{2}\left[\left(N_{0}\right)_{c r}-\left(N_{i}\right)_{c r}\right]}+1\right]^{2}\left[\frac{2 A\left(1-\beta^{2}\right)}{\beta^{2}\left[\left(N_{0}\right)_{c r}-\left(N_{i}\right)_{c r}\right]}-\frac{\left[\left(N_{0}\right)_{c r}-\beta^{2}\left(N_{i}\right)_{c r}\right]}{\beta^{2}\left[\left(N_{0}\right)_{c r}-\left(N_{i}\right)_{c r}\right]}\right.
\end{aligned}
$$

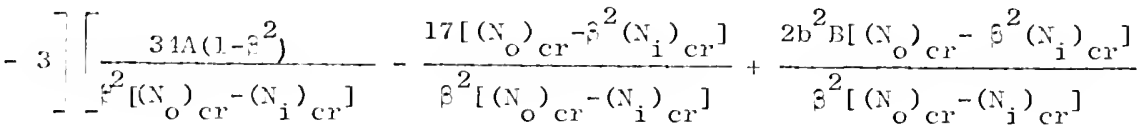

$$
\begin{aligned}
& \left.-2 b^{2} B-1\right]+5\left[\frac{2 A\left(1-\beta^{2}\right)}{\beta^{2}\left[\left(N_{0}\right)_{C r}-\left(N_{i}\right)_{C r}\right]}-\frac{\left[\left(N_{0}\right)_{C r}-\beta^{2}\left(N_{i}\right)_{C r}\right]}{\beta^{2}\left[\left(N_{0}\right)_{C r}-\left(N_{i}\right)_{C r}\right]}+1\right]^{2}
\end{aligned}
$$

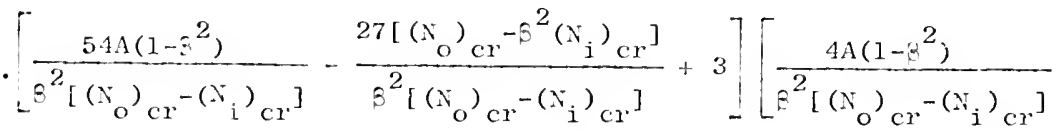

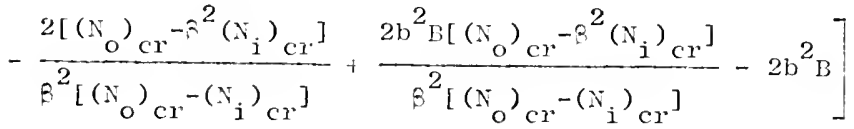

$$
\begin{aligned}
& -5\left[\frac{2 A\left(1-\beta^{2}\right)}{\beta^{2}\left[\left(N_{0}\right)_{C H}-\left(N_{j}\right)_{C r}\right]}-\frac{\left[\left(N_{0}\right)_{C r}-\beta^{2}\left(N_{i}\right)_{C r}\right]}{\beta^{2}\left[\left(N_{0}\right)_{C r}-\left(N_{j}\right)_{C r}\right]}+1\right]\left[\frac{54 A\left(1-\beta^{2}\right)}{\beta^{2}\left[\left(N_{0}\right)_{C r}-\left(N_{i}\right)_{C r}\right]}\right.
\end{aligned}
$$

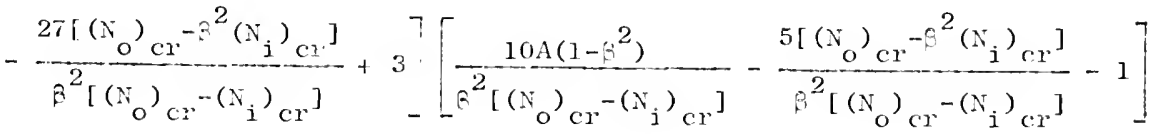




$$
\begin{aligned}
& \left.\cdot\left[\frac{2 A\left(1-\beta^{2}\right)}{\beta^{2}\left[\left(N_{0}\right)_{c r}-\left(X_{i}\right)_{C r}\right]}-\frac{\left[\left(N_{0}\right) \mathrm{cr}^{-\beta^{2}\left(N_{i}\right) c r}\right]}{3^{2}\left[\left(N_{0}\right)_{C r}-\left(N_{i}\right)_{c r}\right]}-3\right]\right\}(\beta-1)^{3}
\end{aligned}
$$

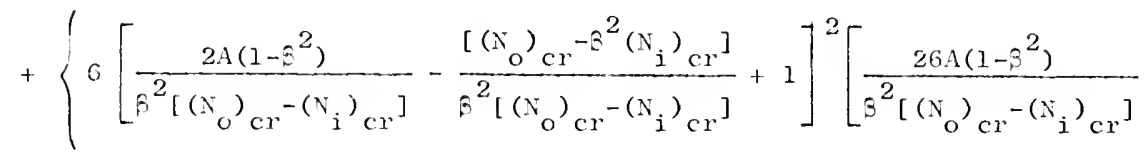

$$
\begin{aligned}
& \left.-\frac{13\left[\left(N_{0}\right)_{C r}-\hat{\beta}^{2}\left(N_{i}\right)_{C r}\right]}{\beta^{2}\left[\left(N_{O}\right)_{C r}-\left(N_{i}\right)_{C r}\right]}+3\right]\left[\frac{2 A\left(I-\beta^{2}\right)}{\beta^{2}\left[\left(N_{O}\right)_{C r}-\left(N_{i}\right)_{C r}\right]}-\frac{\left[\left(N_{0}\right)_{C r}-\beta^{2}\left(N_{i}\right)_{C r}\right]}{\beta^{2}\left[\left(N_{O}\right)_{C r}-\left(N_{i}\right)_{C r}\right]}\right. \\
& \left.+\frac{\delta b^{2} E\left[\left(X_{0}\right)_{C r}-\beta^{2}\left(X_{i}\right)_{C r}\right]}{\beta^{2}\left[\left(N_{0}\right)_{C r}-\left(X_{i}\right)_{C r}\right]}-4 b^{2} B\right]-3\left[\frac{2 A\left(1-\beta^{2}\right)}{\beta^{2}\left[\left(N_{0}\right)_{C r}-\left(N_{i}\right)_{C r}\right]}\right. \\
& \left.-\frac{\left[\left(N_{0}\right)_{C r}-\beta^{2}\left(N_{i}\right)_{C r}\right]}{\beta^{2}\left[\left(N_{0}\right)_{c r}-\left(N_{i}\right)_{C r}\right]}+1\right]\left[\frac{26 A\left(1-\beta^{2}\right)}{\beta^{2}\left[\left(N_{0}\right)_{C r}-\left(N_{i}\right)_{C r}\right]}-\frac{13\left[\left(N_{0}\right)_{c r}-\beta^{2}\left(N_{i}\right)_{c r}\right]}{\beta^{2}\left[\left(N_{0}\right)_{c r}-\left(N_{i}\right)_{C r}\right]}\right. \\
& +3\left[\frac{2 \lambda\left(1-\beta^{2}\right)}{\beta^{2}\left[\left(X_{0}\right)_{C r}-\left(X_{i}\right)_{C r}\right]}-\frac{\left[\left(N_{0}\right)_{C r}-\beta^{2}\left(N_{i}\right)_{C r}\right]}{\beta^{2}\left[\left(X_{O}\right)_{C r}-\left(X_{i}\right)_{C r}\right]} \cdot 3\right]\left[\frac{34 A\left(1-z^{2}\right)}{\beta^{2}\left[\left(N_{0}\right)_{C r}-\left(N_{i}\right)_{C r}\right]}\right.
\end{aligned}
$$

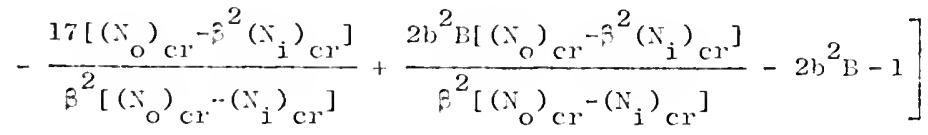

$$
\begin{aligned}
& -\left[\frac{2 A\left(1-\beta^{2}\right)}{\beta^{2}\left[\left(N_{0}\right)_{c r}-\left(N_{i}\right)_{C r}\right]} \cdot \frac{\left[\left(N_{0}\right)_{C r}-\beta^{2}\left(N_{i}\right)_{C r}\right]}{\beta^{2}\left[\left(N_{O}\right)_{C r}-\left(N_{i}\right)_{c r}\right]}+1\right]\left[\frac{26 A\left(1-\beta^{2}\right)}{\beta^{2}\left[\left(N_{O}\right)_{C r}-\left(N_{i}\right)_{C r}\right]}\right. \\
& \left.-\frac{13\left[\left(N_{0}\right)_{C r}-\beta^{2}\left(N_{i}\right)_{C r}\right]}{\beta^{2}\left[\left(N_{0}\right)_{C r}-\left(N_{i}\right)_{C r}\right]}+3\right]\left[\frac{54 A\left(1-\beta^{2}\right)}{\beta^{2}\left[\left(N_{0}\right)_{C r}-\left(N_{i}\right)_{C r}\right]}-\frac{27\left[\left(N_{O}\right)_{C r}-\beta^{2}\left(N_{i}\right)_{C r}\right]}{\beta^{2}\left[\left(N_{O}\right)_{C r}-\left(N_{i}\right)_{C r}\right]}\right.
\end{aligned}
$$

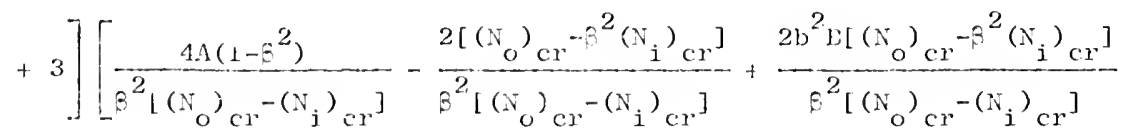


5s:

$$
\begin{aligned}
& \left.-2 b^{2} B\right]+\left[\frac{26 A\left(1-Q^{2}\right)}{\beta^{2}\left[\left(N_{O}\right)_{C r}-\left(N_{i}\right)_{C r}\right]}-\frac{13\left[\left(N_{O}\right)_{C r}-\beta^{2}\left(N_{i}\right)_{C r}\right]}{\beta^{2}\left[\left(N_{O}\right)_{C r}-\left(N_{i}\right)_{C r}\right]}+3\right]
\end{aligned}
$$

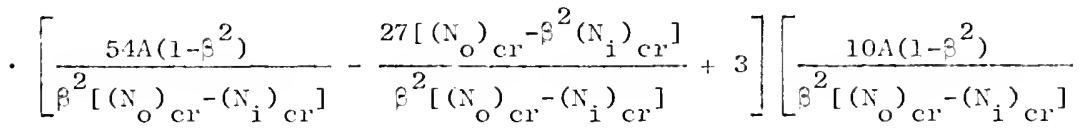

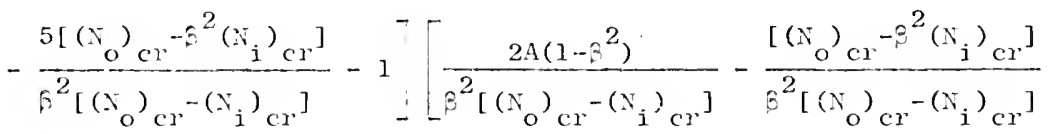

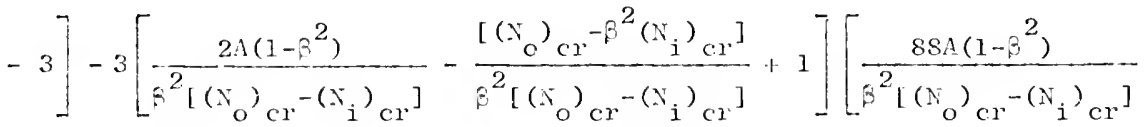

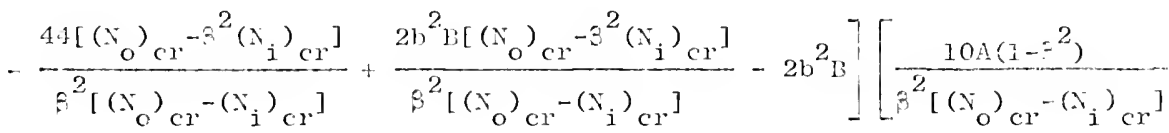

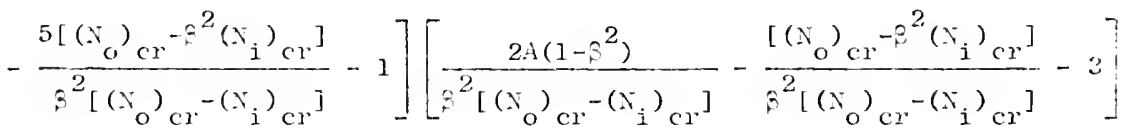

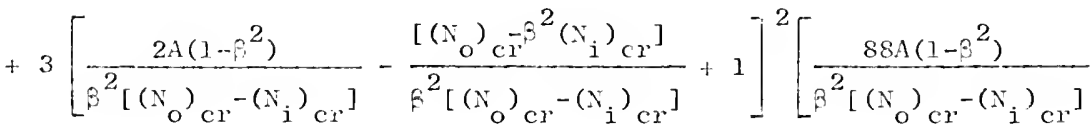

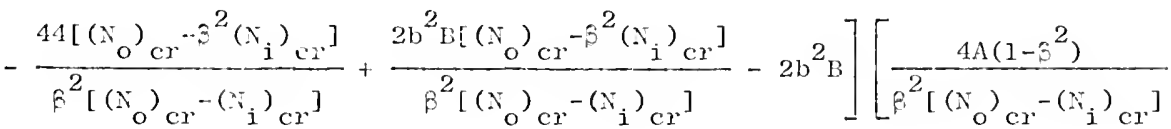

$$
\begin{aligned}
& \left.-\frac{2\left[\left(N_{0}\right)_{C r}-\beta^{2}\left(N_{i}\right)_{C r}\right]}{\beta^{2}\left[\left(N_{O}\right)_{C r}-\left(N_{i}\right)_{C r}\right]}+\frac{2 b^{2} B\left[\left(N_{O}\right)_{C r}-\beta^{2}\left(N_{i}\right)_{C r}\right]}{\beta^{2}\left[\left(N_{O}\right)_{C r}-\left(N_{i}\right)_{C r}\right]}-2 b^{2} B\right]
\end{aligned}
$$

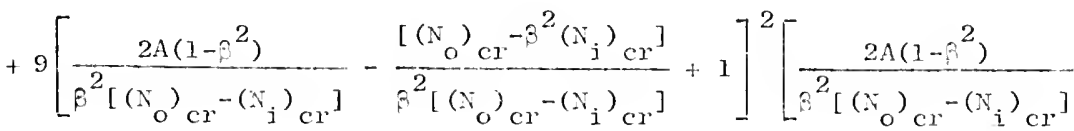




$$
\begin{aligned}
& \left.-\frac{\left[\left(N_{0}\right)_{c r}-\beta^{2}\left(N_{i}\right)_{c r}\right]}{\beta^{2}\left[\left(N_{0}\right)_{c r}-\left(N_{j}\right)_{c r}\right]}-3\right]\left[\frac{24 A\left(1-\beta^{2}\right)}{\beta^{2}\left[\left(N_{0}\right)_{c r}-\left(N_{i}\right)_{c r}\right]}-\frac{12\left[\left(N_{0}\right)_{c r}-\beta^{2}\left(N_{i}\right)_{c r}\right]}{\beta^{2}\left[\left(N_{0}\right)_{c r}-\left(N_{i}\right)_{c r}\right]}\right. \\
& \left.+\frac{8 \mathrm{~b}^{2} \mathrm{~B}\left[\left(\mathrm{~N}_{\mathrm{O}}\right)_{\mathrm{cr}}-^{2}\left(\mathrm{~N}_{i}\right)_{\mathrm{cr}}\right]}{\beta^{2}\left[\left(\mathrm{~N}_{\mathrm{O}}\right)_{\mathrm{cr}}-\left(\mathrm{N}_{\mathrm{i}}\right)_{\mathrm{Cr}}\right]}-4 \mathrm{~b}^{2} \mathrm{~B}\right]-18\left[\frac{2 \mathrm{~A}\left(1-\beta^{2}\right)}{\beta^{2}\left[\left(\mathrm{~N}_{\mathrm{O}}\right)_{\mathrm{Cr}}-\left(\mathrm{N}_{\mathrm{i}}\right)_{\mathrm{Cr}}\right]}\right.
\end{aligned}
$$

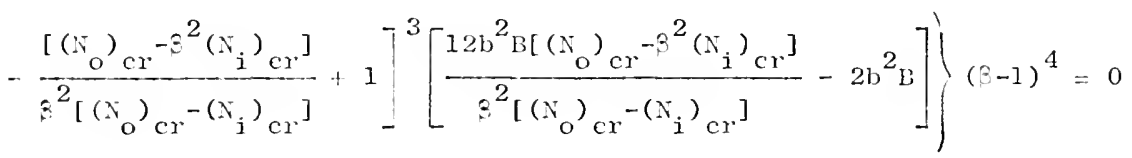

Computation of the sixth approximation is umecessary since it can be shown that the fifth approximation yields acceptable results (see section 5 , Chapter V).

Equations (111-111), each being self-contained, represent the approximate critjcal buckling criteria for an amnular sandwich panel constrained by boundary conditions (74) and (75) and subjecied to uniform radial compressive loads, $\mathrm{N}_{i}$ and $\mathrm{x}_{0}$, along the inner and ouler edges, respectively.

4. Numcrical Results and Discussions

Once the inner compressive lcad, $N_{i}$, is prescribed to be some multiple of the outer compressive load, $N_{0}$, the approximate critical conditions given in the previous section are comletely defined by three dimonsiontess parameters: $\quad\left(N_{0} / A\right)_{c r}, b^{2} B$, and $\beta$. Thus we obtain algewraic polynomials in $\left(N_{0} / A\right)$ which increase in degree as the order. of approxination jnereases (ranging from first dogree in the second approymation to fourth degrec in the fiftn approvination). For 
obvious reasons we consider only the lowest positive value of $\left(\mathrm{N}_{\mathrm{O}} / \mathrm{A}\right)_{\mathrm{cr}}$ satisfying each polynomial.

Three possible loading conditions are analyzed in the present

work: (1) $\mathrm{N}_{0}=\mathrm{N}_{\mathbf{i}}$, (2) $\mathrm{N}_{0}=0$, and (3) $\mathrm{N}_{i}=0$. However, it should be noted that the techniques employed in these examples are applicable for any ratio of $x_{0}$ to $N_{i}$.

$\mathrm{N}_{\mathrm{O}}=\mathrm{x}_{\mathrm{i}}$

If the imer anc outer axial compressive loads are equal, then the exact solution is given by equation (87) in conjunction with Table 2. Thus, the approximation techniques employed in the previous section are umecessary. For this case, Figure 3 shows the existing relation between $\left(X_{0} A{ }_{c r}\right.$ and 3 for various values of $b^{2} \mathrm{~b}$.

$N_{0}=0$

If the inner edge alone is subjected to axial compression, then, since equations (lll-1I4) yield no positive values of $\left(\mathrm{N}_{\mathrm{O}} / \mathrm{A}\right)_{\mathrm{Cr}}$, it can be concluded that bucking never occurs. This would seem reasonable, since, from equations (63-65), such a reduction results in a relatively large tensile $N_{\theta e}$ compared with a rolatively small compressive $\mathrm{N}_{-\mathrm{r}}$. Analogously, for a rectancular single-layer panel subjected to compression along opposite edges and tension along; adjacent edges, Tiroshenko [3] has shown that a large tensile load will prevent a significantly smaller compressive load from causing instability. Howerer, we must keep in mind that, while $\mathrm{N}_{\mathrm{xx}}$ and $\mathrm{N}_{\mathrm{y} y}$ can be varied independently in a rectangular panel, such is not the case 
for a circular or annular panel, since the following equilibriur conclition must be maintained:

$$
\frac{d r}{d r}+\frac{N_{r r}-\underline{N}_{\theta \theta}}{r}=0
$$

$\underline{N_{i}}=0$

If only the outer edge is subjected to axial compression, the second approximation, equation (111), can be solved expljcitly for $\left(x_{0} / A\right)_{C r}:$

$$
\left(\frac{N_{O}}{A}\right)_{c r}=\frac{2(1+3)(3-\hat{\beta})}{3+2 \beta+3 \beta^{2}}
$$

Since $b^{2} z$ does not enter into relation (115), it is obvious that further approximations must be considered.

Dur to the complexity of the calculations involved, a graphical solution is employed for the succecting approximations. In Figre 4 approximations two through five are compared by ploiting $\left(\mathrm{N}_{\mathrm{o}} / \mathrm{A}\right) \mathrm{cr}$ versus $\beta$ for various values of $\mathrm{b}^{2} \mathrm{~b}$.

All quastities appearing in Figures 3 and 4 are dimensionless. Buckling loads may be obtained in the apmopriate dimensions by using tle first of relatjons (73). 


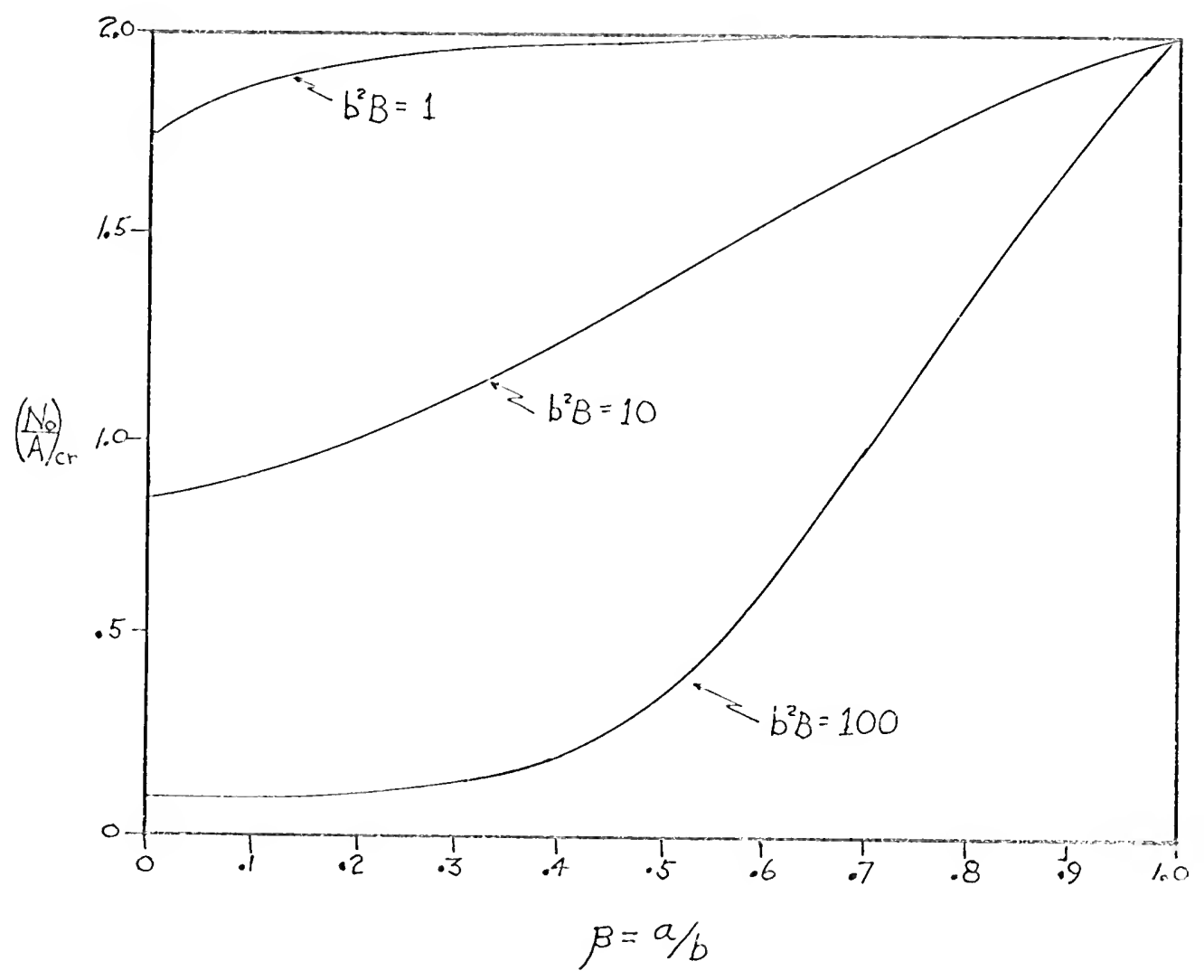

Figure 3. Nirimum Critical values of $\left(N_{0} / A\right)$ for $N_{0}=N_{i}$ 


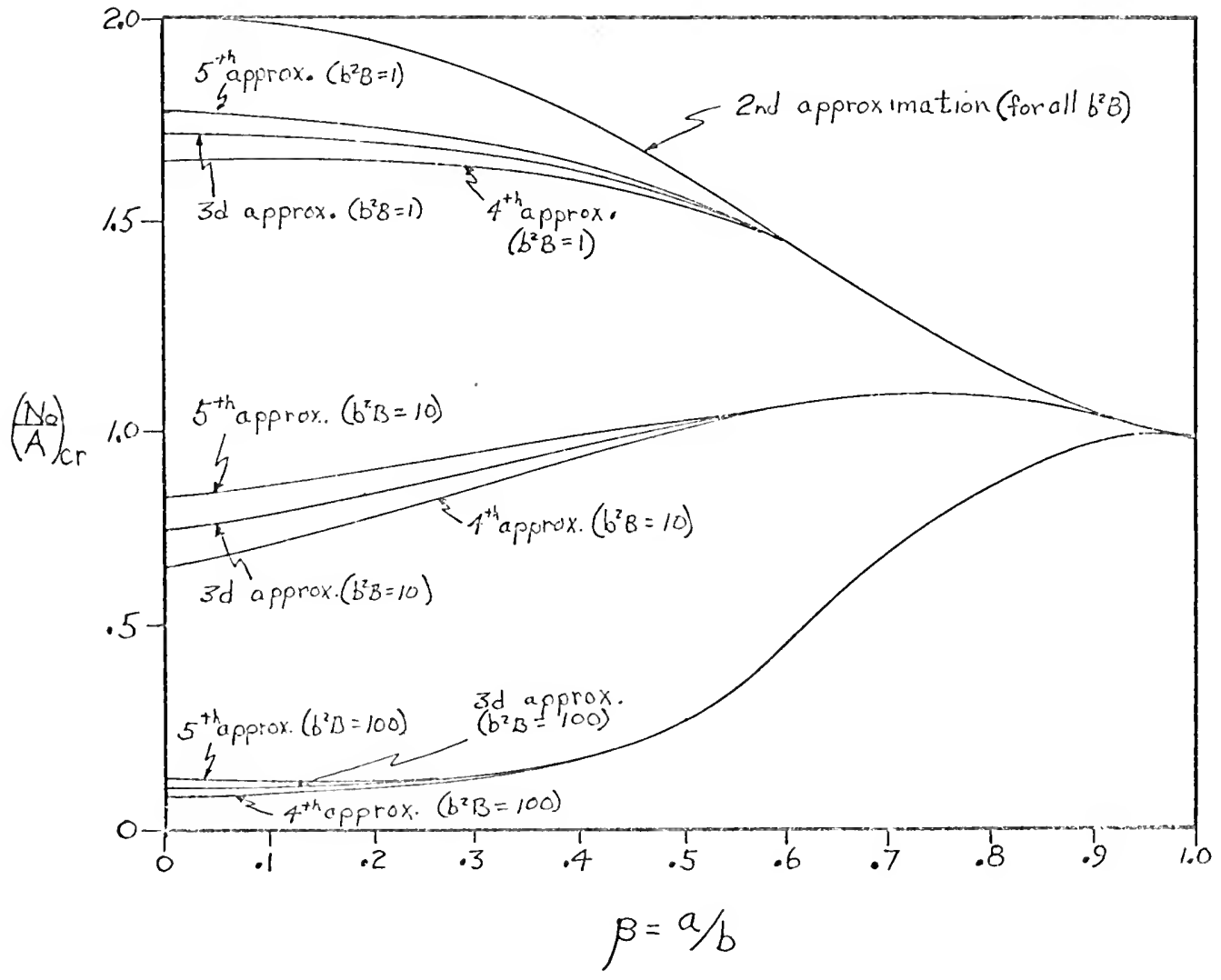

Fisure $A$. Minimum critical values of $\left(N_{0} / A\right)$ for $N_{i}=0$ 
The following conclusions may be drawn from figures 3 and 4:

1. All critical values of $\left(\mathrm{N}_{\mathrm{O}} / \mathrm{A}\right)$ are less than or equal to two. (Figures 3 and 4)

2. As the value of $b^{2} B$ approaches infinity, the results approach those obtained for a single-1ayer panel in equation (89). (Figure 3)

3. For $\beta=0$, the present theory coincides with Huang and Ebeioglu's results (equation (88)) for a circular sandich panel. (Figures 3 and 1)

4. The second approxination in Figure 4 is the exact solution for $\mathrm{b}^{2} \mathrm{~B}=0$.

5. An anmular sandwich panel subjected to axial compression atong the outer boundary becomes stronger if an equal compressive load is also applied along the inner edige. (Figures 3 and 4 )

6. In Figure 1 , the third approximations yield more accurate result: than the fourth approxinations. This peculjarity and the error bound associated with the fifth approximations will be discussed in the next section.

7. A dual response is apparent in Figure 4. As the hole increases in relative size, the panc] may become weaker or stronger depending on tl:e value of $\mathrm{b}^{2} \mathrm{~B}$ and the range of $\beta$ being considered. Such a behavior is possible because both the shear and the bending stiffness of a sandwich panel enter into the analysis. An amular single-iayer panel, which can be described by only two dimensionless perameters, exhibits no such dual response. 
- 8. If bucking loads for values of $b^{2} \mathrm{~B}$ or ratios of $\mathrm{N}_{0}$ to $\mathrm{N}_{i}$, not considered in Figures 3 and 4 , are required, equation (J14) mey be used directly. However, when considering only values of $f$ greater than one-half, the third approximation, equation (112), yields acceptable results. (Figure 4)

The above conclusions are valid only for the special case in which boundary condjtions (74) and (75) are applied.

\section{Error Bc.und}

Since ve chose to expand the solution of equation (93) about the point it =- 1, the speed of convergence of the scries solution obtained, equation (10\%), depends on the proximity of the entire ammlar rewion to that point. Cleurly, as the hole increases in size (5 approaches one), the solution converges more rapidly. This fact is cvident fron rigure 4, and also from equation (107). Indeed, it can be conciuded that the speed of convergence is the slowest when $\delta=0$.

Frch equations (111-114) the approximate critical bucking paraneter, (N, A) ${ }_{\mathrm{CH}}$, can be solved explicitly for the degcrerate cast of $\hat{\theta}=0$. The results of this simplification are found in Table 3 . However, fow this special case, the exact solution (reproducti here for convenience) is available from section 1 of this chapter:

$$
\left(\frac{\mathrm{N}}{\mathrm{A}}\right)_{\mathrm{cr}}=\frac{14.681}{\mathrm{~L}^{2} \mathrm{~B}+7.312}
$$


TABLI 3

$$
\begin{gathered}
\text { APPROXINATE VALUES OF }\left(N_{0} / A\right) \mathrm{cr} \\
\text { FOR } \beta=0
\end{gathered}
$$

Orcier cf

Approxination

$$
\left(\mathrm{N}_{\mathrm{O}} / \mathrm{A}\right) \mathrm{cr}
$$

\begin{tabular}{|c|c|}
\hline First & (No results) \\
\hline Seconci, equation (111) & 2 \\
\hline Thira, equation (112) & $\frac{12}{\mathrm{~b}^{2} \mathrm{~B}+6}$ \\
\hline Fourth, cquation (113) & $\frac{10}{\mathrm{~b}^{2}+5}$ \\
\hline Fifth, equation (114) & $\frac{15}{\mathrm{~b}^{2} \mathrm{~B}+7.5}$ \\
\hline
\end{tabular}

If we compare cquation ( 88$)$ with Table 3 , it can be concluded that the fifth appoximation, for $\beta=0$, is within 2.2 per cent of the exact solution, even for large values of $\mathrm{b}^{2} \mathrm{~B}$. And, since the series solution converges more rapidly for other values of $\beta$, equation (114) yielcis results that lie within 2.2 per cent of the exact solution for all values of $b^{2} 1$; and 3 .

Following the same rcasoning outlined above, it can be conclucied that the third approximation yields nore accurate results than the fourth approximation. This peculiarity can be easily verified by expanding a series solution of ecuation (78) about the point $r=b$ $(\xi=0)$. If this is done, the rcsulting approximations, as expected, coincide exactly with thosc listed in Table 3. 


\section{Remarks on Convergence}

The serjes solution of an ordinary differential equation possesses a radius of convergence at least as great as the distance from the point of expansion to the nearest singularity [19].

Equation (93) possesses two regular singularities, one at $\eta=0$, and another at $\left(\mathrm{N}^{2}+1\right)=0$; and its solution was expanded about the point $\eta=1$. Therefore, it must be demonstrated that these singularities do not inhibit the validity of our solution throughout the entire anmular region of the pancl.

Referring to Figure 5 , it becomes obvious that the singularily at $\eta=0$ does not restrict the required radius of convergence, regardless of the value of $\hat{\sigma}$. It is therefore necessary only to show that the singularity occurring at $\left(N^{2}+1\right)=0$, lies outside the amular region and its reflection illustrated in Figure 5, for all values of 6 .

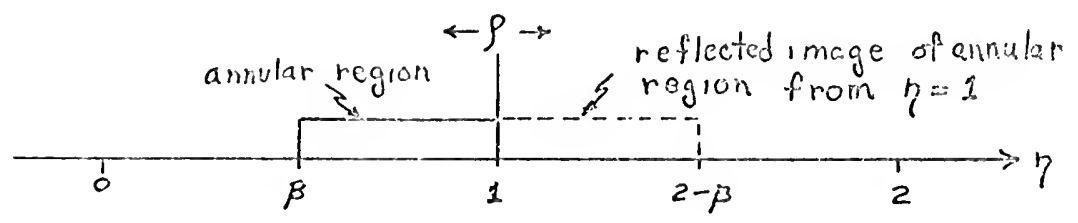

Figure 5. Radius of Convergence

With the aid of cquation $(108),\left(\mathrm{N}^{2}+1\right)=0$ becomes:

$$
\frac{r_{1}^{2}}{\beta^{2}\left(N_{0}-n_{i}\right)}\left[2 A\left(1-\beta^{2}\right)-\left(N_{0}-\beta^{2} N_{i}\right)\right]+1=0
$$


Therefore, the position of the sirgularity associated with equation (116) depends on the value of the critical buckling load which becomes known only after the solution is obtained.

For the particular case in which $N_{i}=0,(116)$ is satjsfied if

$$
\left(\frac{N o}{\bar{A}}\right)_{c r}=\frac{2(1-\hat{3})^{2}}{\eta^{2}-\beta^{2}}
$$

Howerex, from equation (115) and Figure 4 , it is appaxent that, for $:_{i}=0$,

$$
\left(\frac{N o}{A}\right)_{C r} \leq \frac{2(1+\beta)(3-\beta)}{3+2 \beta+3 \beta^{2}}
$$

for all values of $b^{2}$ is.

In ondcr that conditions (117) and (118) be satisfied simultancously, $r^{2}$ must satisfy the following inequality:

$$
\eta^{2} \geq \frac{2(3-\beta) \beta^{2}}{\left[2(3-\beta)-2(1-\beta)\left(3+2 \hat{\beta}+3 \beta^{2}\right)\right]}
$$

Equation (119) constrains $\eta^{2}$ to be greater than $(2-3)^{2}$ for all values of $\beta$ ranging from 0 to 1 . It can therefore be concludcd that the singularity associated with equation (117) lies outside the annular region and its reflection illustrated in Figure 5. Similarly, it can also be shown that, for $\mathrm{N}_{\mathrm{O}}=0$, the singularity associated with equation (1.16) lies outside this eritjcal region. 
Thus, the radius of convergence, $\rho$, of the series solution of equation (93), cxpanded about the point $\eta=1$, is:

$$
\rho \geq 1-\beta
$$

which, as illustrated in Figure 5, is large cnough to encompass the entire annular rerion of the panel.

Care must be taken, however, when imposing boundary conditions other than (74) and (75) on the scilution of equation (93). Critical loads resulting from boundary condjtions or ratios of $N_{0}$ to $N_{i}$ not considered in the present work maj satisfy equation (116) within the needed radius of convergence. It would then become necessary to either. expand the sclution of equation (93) about some other point, or employ the techniques associated with analytic continuation. Tne difficulties encountered in the latter approach would be enormous. 


\section{CHAPTER V1}

\section{CONCLUSION}

The present work investigates the buckling of annular sandwich panejs. Equilibritm equations and boundary conditions satisfying continuity requirements were derived in cartesian coordinates, using the theoren of minimum potential energy. These equations were then transformed into polar coordinates through the application of tensnr analysis.

Axisymetric buckling being assmed, and the bending rigidity of the faces being neslected, the courlibrium equations were uncoupled by using a nodifjed technique. The governing equations were then compared with exising theories for single-layer annular panels []1,12] and circular sandwich pane]s [10].

For the general problem of an amular sandwich panel subjected to unequal inner and outer compressive loads, and constrained by boundary corditions similar to those employed by Oisson [12], a power series solution was obtained. This series was shown to possess a radius of convergerce of sufficient magnitude. Successive approxinations vere then computed, and a graphical solution was employed for various ratios of outer to inner compressive loads. Results from the fifth approxination, which were shown to be within 2.2 per cent of the exact solution, were compared with ibose obtained from earlier theories $[10,11,12]$ 
The present work represcnts the first aitempt to analyze the stability of annular sandwicl panels. Further extensions of the present theory nay be carried out by including the effects of the bending rigidity of the faces or considering boundary conditions other than those employed here. Furthermore, continued efforts should be directed toward obtaining a solution to the unsymetric buckling problem. In this way, the assumption of axisymetric buckling coud be justified, and the problems associated with angular dependent loading functions could be analyzed. 


\section{BIBLIOGRAPIIY}

1. F. J. Plantema, "Sandwich Construction," Wiley and Sons, New York, 1966.

2. S. Timoshenlio and S. Woinowsky-krieger, "Thoory of Plates and Shel1s," 2nd ed., McGraw-l1il1, New York, 1959.

3. S. Timoshenko and J. Gere, "Theory of Elastic Stabjlity," 2nd ed., McGraw-Hill, New York, 1961.

4. N. J. lioff, "Bencling and Buckling of Rectangular Sandwich plates," NACA TN 2225, 1950.

5. A. C. Eringen, "Bending and Buckling of Rectangular Sandwich plates," Proc. First U.S. Natl. Cong. Appl. Nech., 1951, pr. 381-390.

6. C. C. Chang and 1. K. Ebcioglu, "Elastic Instability of Rectangular Sandwich panei of Orthotropic Core with Different Face Thicknesses and haterials," Transactions of the Anerican Society of Mechanical Engineers, J. App. Mech., Vol. 27, No. 3, Septenber i $960, \mathrm{pp} .474-480$.

7. S. J. Kim, "Symmetrie and Antisymetric Buckling of Sandwicir pancls," loctoral Dissertation, Dept. Eng. Sci. and Mech., University of Florida, 1969.

8. F. Reissner, "Small Bending and Stretching of Sandwich-Type Shells," NACA TN $1832,1949$.

9. M. Zaid, "Symmetrical Benling of Cjrcular Sandwic! plates," Mve. of the 2nd U.S. Nat1. Cong. Appl. Mech., 1951, pp. 413-122.

10. J. C. lluang and I. K. Ebuigglu, "Circular Sandwich plate under" Radial Compicssion and Thermal Gradient," AIAA Journal, Vol. 3, No. 6, June 1565, pp. 1146-1148.

11. F. Meissner, "Uher das Knicken kreisringförmiger Scheihen," Schweiz. Biturg., Vol. 101, 1933, pp. 87-89.

12. G. O1sson, "Knichung der krejsringlatte von quadratisch verändexlicher Steifigheit," Ingr.-Arch., Vol. 9, 1938, pp. 205214. 
13. C. C. Chang and I. K. Elicioglu, "Thermoclastic Eehavior of a Simply Supported Sendwion Pane] Under Large Temperature Gradient and Exige Compression," J. Aero. Sei., Vol. 28, No. 6 , June 1961 , pp. $480-192$.

14. I. S. Sokolnikoff, "Mathematical Theory of Elasticity," 2nd ed., NoGraw-Hill, New York, 1956.

15. Y. C. Fung, "Foundations of Solid Mechanics," Prentice-lall, Englewood Cliffs, New Jersey, 1965.

16. A. J. McComell, "Applications of Tensor Analysis," Dover Publjcations, Inc., New York, 1957.

17. S. Timoshenko, "Strength of Naterials," Part II, D. van Nostrand, princeton, New Jersey, 1956.

18. F. F. Hildebrand, "Advanced Calculus for Apllications," PlenticeHal1, Lnglewond Cliffs, New Jersey, 1962.

19. I. R. Ford, "Differential Equations," 2nd ed. , Mckraw-Hil], New York, 1955. 


\section{BIOGRAPHICAI, SKETCH}

Ameljo John Anato was born in Newark, Now Jersey, on Jankary 20, 19.14. He was graduated from Seton Hall preparatory School in June, 1962. In June, 1966, he received the degree of Baclielor of Science in Nechanical Engineering from Newark College of Engineering (xew Jersey).

In September of the same year he entered the Department of Enginecring Science and Nechanics at the Unjversity of Florida as a National hefense and Education Act, Title IV Fellow. Here, in August, 1967, he received the dogree of nister of science in Fngineering prior to pursuing the degree of noctor of Philosophy. 
This dissertation was prepared under the direction of the chairman of the candidate's supervisory committee and has been approved by all members of that comittee. It was sumittod to the Dean of the College of Ingincering and to the Graduate Council, and was approved as partial fulfilment of the requirements for the degree of Doctor of Philocophy.

June, 1970

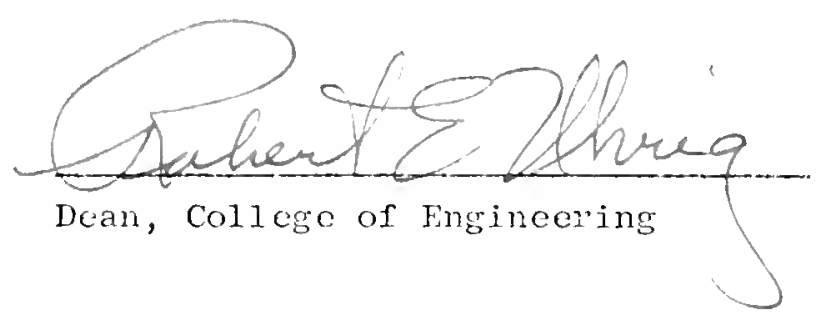

Dean, Graduate School

Supervisory Comnittee:
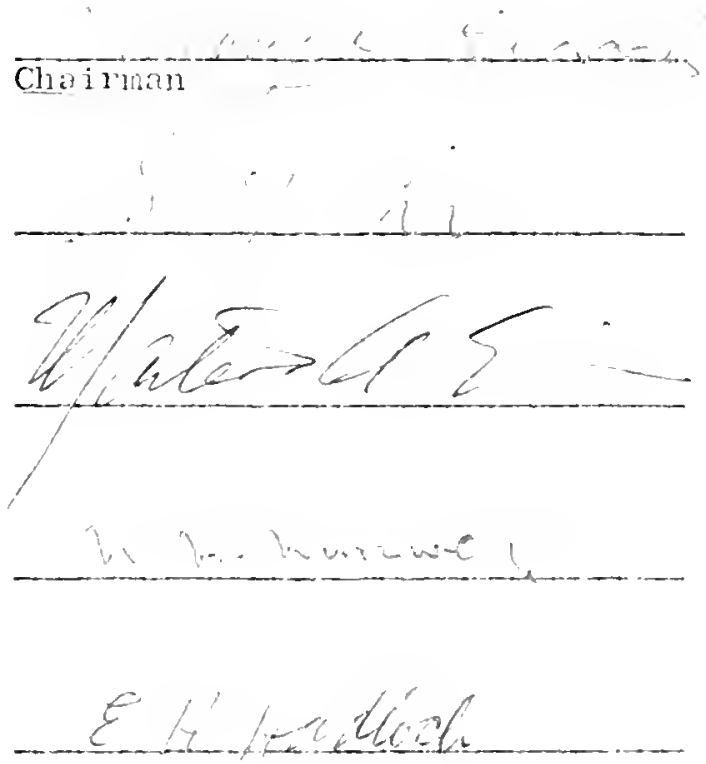


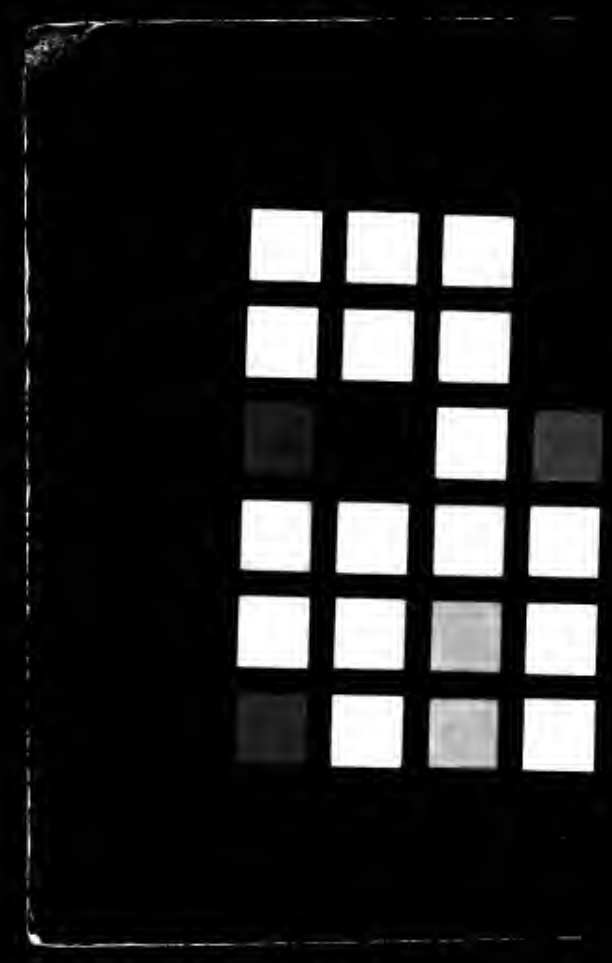



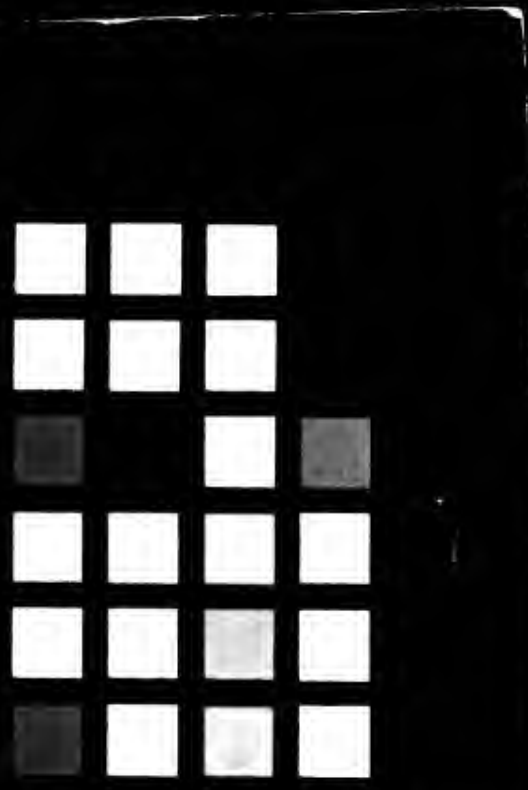